\title{
An assessment of the antidepressant-like properties of erythropoietin in an animal model of depression
}

\author{
by
}

Kyla Vanderzwet

A thesis submitted to the Faculty of Graduate and Postdoctoral Affairs in partial fulfillment of the requirements for the degree of

\author{
Master of Science \\ in \\ Neuroscience \\ Carleton University \\ Ottawa, Ontario
}

May 2015

(C) 2015

Kyla Vanderzwet 


\begin{abstract}
Impaired neuroplasticity and altered connectivity between different brain regions may be key features of depression. As the cytokine erythropoietin (EPO) increases brain derived neurotrophic factor (BDNF) levels, EPO may enhance neuron survival and growth, leading to antidepressant effects. Here, EPO was tested for its ability to reverse depressive-like symptoms in rats following three weeks of chronic mild stressors. Neither stressor exposure nor EPO affected sucrose preference, which has been used to model the anhedonia characteristic of depression. Additionally, EPO did not reverse stressor-induced social avoidance. BDNF mRNA expression was reduced by stressor and EPO treatments in the prefrontal cortex, and elevated in the amygdala with EPO treatment. Additionally, FGF-2 expression was reduced in the amygdala with stressor exposure, but normalized following EPO treatment. Many of the results are inconsistent with a priori hypotheses, possibly owing to factors such as the timing of tissue collection and a lack of environmental enrichment.
\end{abstract}




\section{Acknowledgements}

I would like to express my gratitude to my supervisor, Hymie Anisman, for his supportive guidance throughout this degree. I could not have hoped for a better supervisor. I also extend my thanks to Shawn Hayley for overseeing this project, and to Alfonso Abizaid, for acting on my thesis advisory committee along with Dr. Anisman and Dr. Hayley. Thank you to Iain McKinnell and Kim Hellemans as well, for taking part in my thesis defense.

I'd also like to acknowledge and thank Jon Constable, Nils Gritters, and Krystin Elson for their roles in completing all of the lab work. Furthermore, many thanks go to Marzena Sieczkos, Teresa Fortin, and Kyle Farmer for their help and instruction along the way. It was a team effort - thanks for making it possible! 


\section{Table of Contents}



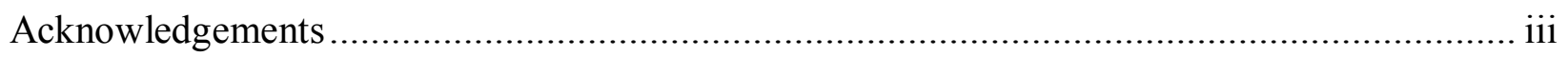

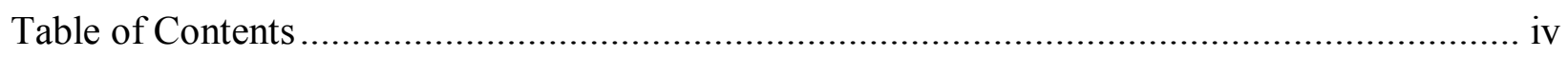

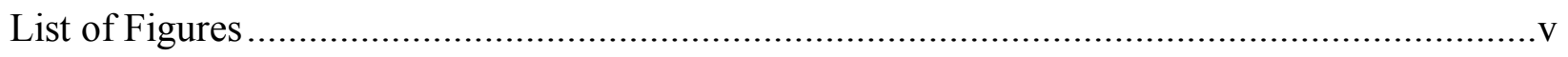

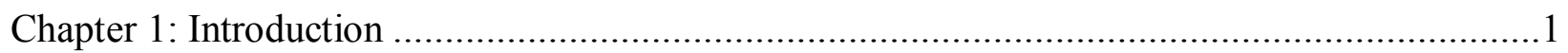

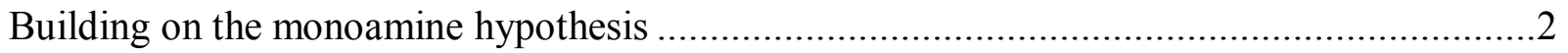

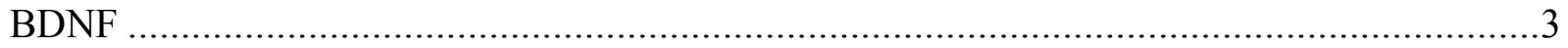



Glucocorticoids and growth factor levels impact neurogenesis and neuronal architecture ........6

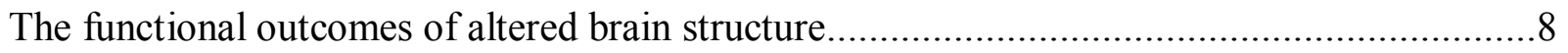



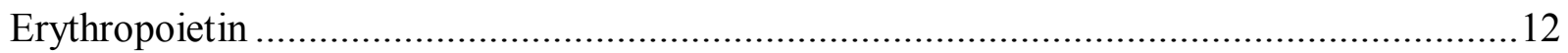

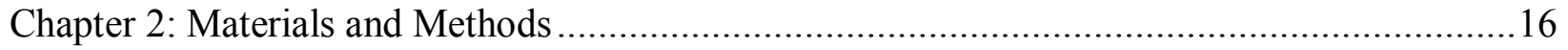

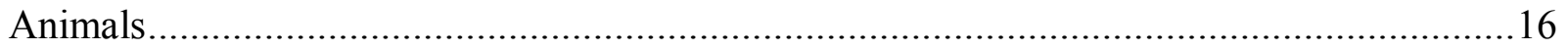

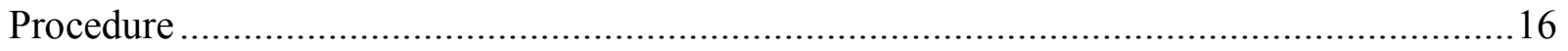

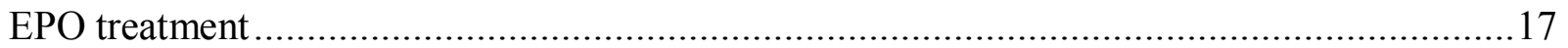

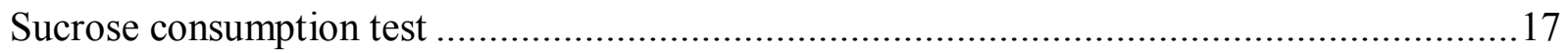



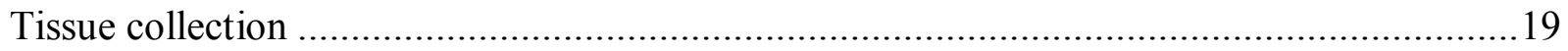

Reverse transcription-quantitative polymerase chain reaction analysis (RT-qPCR) ................20

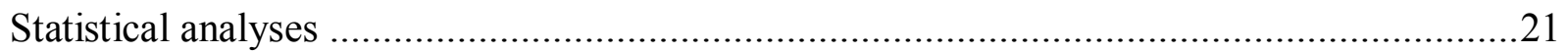





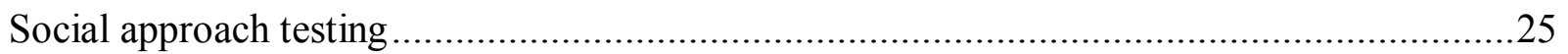

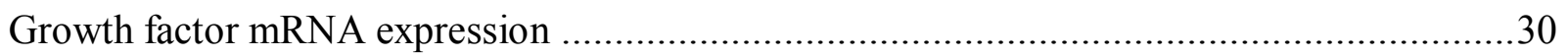

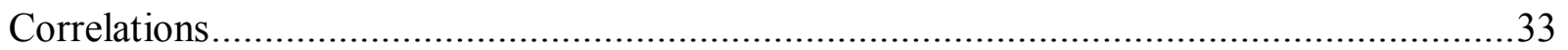





Social avoidance in a three-chambered social approach test....................................................38

Changes in growth factor expression in relation to stressor conditions ..................................42

Changes in growth factor expression in relation to EPO treatment .........................................4



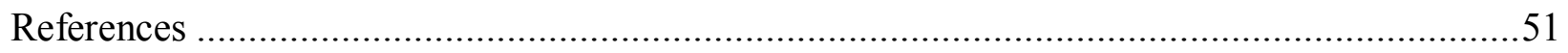




\section{List of Figures}

Figure 1: Results of the sucrose consumption test.......................................................23

Figure 2: Preference for a 1\% sucrose solution in individual animals..................................24

Figure 3: Results of social approach testing............................Error! Bookmark not defined.

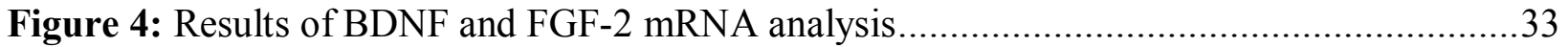




\section{Chapter 1: Introduction}

Depression involves changes of brain structure and function, one of which is a reduction in hippocampal volume, likely attributable to impaired neurogenesis and dendritic branching as a result of stress (Duman, 2014). Moreover, hippocampal volume was negatively correlated with the duration of the disease and the number of depressive episodes experienced (Colla et al., 2007; McQueen et al., 2003). Animal models of depression indicated that chronic unpredictable stressors or corticosterone treatments caused impairments of hippocampal neurogenesis and plasticity (Malberg, 2004; Son et al., 2012), and altered connections between the hippocampus and other brain regions could lead to disrupted regulation of moods and emotions in humans (Duman, 2014).

A neuroplastic perspective of depression is supported by the observation that conditions which reduce neurotrophic factors, thereby reducing neuronal survival and growth, promote depressive-like behaviours in animals. Depression-promoting events include physical and social stressors, as well as exposure to corticosterone in rodents. Such treatments can reduce hippocampal levels of brain derived neurotrophic factor (BDNF), which is reversible or preventable with antidepressant treatment (Duman et al., 1999; Dwivedi et al., 2006; Masi \& Brovedani, 2011). Likewise, serum and plasma BDNF levels were reduced in depressed patients (Piccinni et al., 2008), and antidepressant treatment resulted in the normalization of plasma BDNF levels (Piccinni et al., 2008).

In addition to BDNF, basic fibroblast growth factor (FGF-2) is another growth factor influenced by stressors which may be relevant to depression. For example, hippocampal levels of FGF-2 are elevated in response to acute stressors or corticosterone treatment (Molteni et al., 
2001). There was also an increase in FGF-2 mRNA in the hippocampus in response to chronic restraint (Fumagalli et al., 2005). Moreover, repeated treatment with the antidepressants desipramine, fluoxetine, and mianserin all increased hippocampal protein and mRNA levels of FGF-2 (Mallei et al., 2002).

Elevated BDNF or FGF-2 could potentially have antidepressant effects, but some peptide growth factors, notably BDNF, do not appreciably cross the blood-brain barrier due to a high molecular weight and hydrophilic nature (Thorne \& Frey, 2001). Conveniently, the cytokine erythropoietin (EPO) crosses the blood-brain barrier and increases BDNF levels and synthesis (Hayley \& Littlejohn, 2013). As such, EPO may enhance neuron survival and growth, while also having antidepressant effects. Consistent with this view, EPO treatment has consistently led to antidepressant-like effects in mice, rats and humans (Girgenti et al., 2009; Miskowiak et al., 2007; Osborn et al., 2013). Moreover, it had beneficial effects on neuronal proliferation and architecture in vitro (Hoon et al., 2012). Based on this evidence, and consistent with a neuroplastic theory of depression, there is reason to believe that EPO might be useful as an antidepressant or as an adjunct treatment. The present investigation was conducted to assess the effects of EPO on depressive-like behaviours and brain growth factor levels in an animal model of depression.

\section{Building on the monoamine hypothesis}

Most current antidepressant medications act by elevating synaptic levels of serotonin, dopamine and/or norepinephrine. This occurs either by blocking the reuptake or inhibiting the catabolism of these monoamines (Duman, 2004). Based on these modes of action, the commonly cited monoamine hypothesis of depression evolved, but this position can be discounted for several reasons. For example, it has not been consistently demonstrated that 
depressed patients have altered monoamine levels in their plasma, urine, cerebrospinal fluid or brain. Additionally, antidepressants raise monoamine levels immediately, whereas effects on mood only occur two to three weeks after treatment initiation. Finally, current drug treatments are ineffective in a large proportion of patients (Branchi, 2011), and even when effective, symptoms are not entirely eliminated and recurrence of the illness is frequent (Shelton \& Hollon, 2012).

Although the monoamine hypothesis of depression may not be complete, the downstream effects of these drugs could provide clues to the cause of the disease. What serotonin and norepinephrine acting agents are known to do is increase the expression of brain-derived neurotrophic factor $(\mathrm{BDNF})$, its receptor tropomyosin receptor kinase $\mathrm{B}(\operatorname{TrkB})$, and the transcription factor CREB, or cyclic AMP response element binding protein (Chen \& RussoNeustadt, 2013). As will be discussed shortly, this growth factor and its signalling components have been strongly implicated in depression, and the evidence seems to point to disrupted neuroplasticity as a cause of depression.

\section{BDNF}

Growth factors in the brain are important for cellular proliferation, migration, differentiation, and maintenance (Masi \& Brovedani, 2011). BDNF is perhaps the most commonly studied neurotrophic factor in depression research, and is believed to modify neural networks in an experience-dependent way to influence mood (Castrén et al., 2007). BDNF first received attention when it was found to be reduced in the CA3 pyramidal cell layer of the hippocampus and the granule cell layer of the dentate gyrus following single or repeated restraint stressor treatments (Duman, 2004). This observation was made in association with several 
different stressors, including footshock, early maternal stress, and unpredictable physical stressors; and early life stressors likewise reduced BDNF levels in adulthood (Duman, 2004). In addition, studies in humans showed that blood and brain levels of BDNF were reduced in depressed patients, but were normalized by antidepressant treatment (Castrén et al., 2007; Piccinni et al., 2008). Reductions in BDNF and its receptor TrkB were also observed in both the hippocampus and PFC of depressed individuals (Yu \& Chen, 2011), although other research indicated that these reductions are gender-specific (Hayley et al., 2014).

As a mechanism for this change, chromatin remodelling was involved in hippocampal BDNF down-regulation following a social defeat stressor in mice. Tsankova et al (2006) observed a nearly three-fold reduction in BDNF mRNA levels following chronic social defeat, and this was reversed by chronic treatment with imipramine. In addition, these authors found a greater than four-fold increase in repressive histone dimethylation at two different BDNF promoters following chronic defeat, which was not reversed by imipramine treatment. Imipramine treatment did, however, lead to hyperacetylation of histones in BDNF promoter regions, having an activating effect on BDNF expression, but only in previously defeated mice. Imipramine treatment also led to reductions in mRNA levels of histone deacetylase (HDAC)5, whereas, overexpression of HDAC5 blocked the antidepressant-like effect of imipramine in stressed mice. Evidently, chromatin remodelling is one mechanism that leads to reduced BDNF levels following stressor exposure.

Although much of the literature focused on the effects of stressors on hippocampal BDNF alterations, stressor exposure also influenced BDNF expression within the PFC. For example, repeated intermittent social stressor treatment in rats led to a transient increase of 
BDNF protein and mRNA levels in the PFC (Fanous et al., 2010). It was also observed that in the anterior cingulate, which processes emotional information and is involved in decision making, both escapable and inescapable stressors increased BDNF expression, more so following the escapable stressor, likely reflecting an increase of coping responses that would be emitted (Bland et al., 2007). Yet, prenatal and early life stressors reduced BDNF expression in the PFC when assessed in adulthood (Yu \& Chen, 2011). Plus, reduced BDNF levels are observed in the PFC of depressed brains post-mortem (Karege et al., 2005). So, if BDNF elevation in the PFC is part of a coping response shortly after stressor exposure, perhaps early life stressors and the ensuing depression impair or overwhelm this coping mechanism.

The amygdala is yet another brain region in which stressor exposure, such as a chronic intermittent social challenge, generally reduces BDNF protein levels (Fanous et al., 2010). Curiously, when assessed 28 days later this effect disappeared in the basolateral and central amygdala, but BDNF protein levels actually increased in the medial amygdala. In contrast, this study observed an increase in mRNA expression of BDNF in the basolateral and central amygdala 2 hours after stressor termination (Fanous et al., 2010). Elsewhere, a decrease in BDNF mRNA in the basolateral amygdala was observed 24 hours after an acute social defeat stressor (Pizarro et al., 2004). Additionally, one week after chronic unpredictable mild stressor exposure, BDNF protein levels were reduced in the lateral amygdala in rats (Zhang et al., 2014). In humans, alterations in amygdalar BDNF levels have been variable. In post-mortem brain analyses levels of BDNF in the basolateral and central amygdala did not significantly differ between controls, depressed patients that died by suicide, or depressed individuals who died through ways other than suicide, although there was a trend towards increased BDNF in the central amygdala in the latter instance (Maheu et al., 2013). However, both mRNA and protein 
levels of BDNF were reduced in the amygdala of depressed women compared to matched controls (Guilloux et al., 2012).

\section{FGF-2}

In addition to BDNF, basic fibroblast growth factor (FGF-2), is another growth factor whose levels are altered by stressors. FGF-2 is mainly expressed by astroglia, and it promotes the survival and maturation of neurons. It can also stimulate adult neurogenesis (Molteni, 2001). FGF-2 levels are altered by stressor exposure and in depression, but the pattern of changes differs somewhat from that observed with BDNF. For example, following acute stressor exposure the mRNA levels of this growth factor are increased in some brain regions, including the PFC and hippocampus. This effect was greater and more rapid when the stressor was controllable compared to uncontrollable, again suggesting that active coping may limit the effects of a stressor by elevating this growth factor (Bland, 2007). Furthermore, acute and chronic stressor exposure also resulted in an increase in FGF-2 levels in the hippocampus and entorhinal cortex (Fumagalli et al., 2005). Again, this could be an adaptive mechanism activated in response to stress. However, in this study mRNA levels of FGF-2 were reduced in the PFC following chronic stressor exposure (Fumagalli et al., 2005). Additionally, levels of FGF-2 and its receptors were reported to be reduced in the PFC of depressed humans (Evans et al., 2004). So if PFC levels of FGF-2 are elevated as an adaptation to acute stressors, perhaps this leads to depleted levels on a long-term scale.

\section{Glucocorticoids and growth factor levels impact neurogenesis and neuronal architecture}

Chronic stressors may lead to impaired hippocampal neurogenesis. This occurs in the subgranular zone of the dentate gyrus, where progenitor cells divide and migrate to the granular 
cell layer and hilus. The rate at which this occurs depends on glucocorticoid concentrations (Duman et al., 1999). Both chronic and acute stressors of variable nature have neurogenesissuppressing effects across several species (Mirescu \& Gould, 2006). Whereas environmental enrichment is associated with increased neurogenesis and improved learning, reduced neurogenesis may contribute to impaired hippocampal function (Duman et al., 1999).

Reduced dendritic branching in the hippocampus is another side-effect of chronic stressor exposure (McEwen, 2004). For example, one month of chronic unpredictable stressors or daily subcutaneous injections of corticosterone $(40 \mathrm{mg} / \mathrm{kg}$ ) reduced dendrite outgrowth in the CA3 hippocampal region and resulted in atrophy in granule and CA1 pyramidal cells (Sousa et al., 2000). Generally, reduced size and function of the hippocampus occurs in diseases marked by elevated glucocorticoids. This includes depression, PTSD, and Cushing's disease, and also in elderly people with elevated cortisol levels (Colla et al., 2007; Duman et al. 1999).

In addition to altering the structure of the hippocampus, elevated glucocorticoids and diminished growth factor levels affected the structure of the prefrontal cortex. For example, chronic corticosterone exposure led to reduced expression of neural cell adhesion molecules in the frontal and prefrontal cortex, suggesting that glucocorticoids may alter synapse stability and neuronal structure in these regions (Sandi \& Loscertales, 1999). Furthermore, chronic restraint led to apical dendrite retraction and debranching, and axospinous synapse loss in the medial prefrontal cortex (mPFC) (Liston et al., 2006). Likewise, chronic subcutaneous injections of corticosterone $(10 \mathrm{mg})$ in rats led to a reorganization of the dendrites of pyramidal neurons in layers II-III of the mPFC, so that there was more dendritic material near the cell body and less in distal regions (Wellman, 2001). Although this could be due to the direct action of corticosterone 
in this region, the reorganization could also have been due to reduced inputs from the hippocampal CA3 pyramidal neurons. Pyramidal neurons in the $\mathrm{mPFC}$ primarily receive extracortical inputs at distal dendrites, and cortical inputs on proximal dendrites. Therefore, proximal dendritic material may have been increased to amplify and compensate for reduced distal dendritic inputs from the hippocampus (Wellman, 2001). This switch to increased intracortical signalling and reduced subcortical connectivity would likely contribute to the cognitive effects of stress (Wellman, 2001).

The structural effects of stressors also arise in the amygdala. For example, although 10 days of chronic immobilization reduced hippocampal CA3 dendritic branching, arborisation was increased in stellate and pyramidal neurons of the basolateral amygdala (Vyas et al., 2002). Paralleling these changes, hyperactivation of the amygdala was associated with depression (McEwen, 2004). Given that the amygdala activates the HPA axis, this amygdalar hyperactivation could contribute to the excessive glucocorticoid release seen in depression (Vyas et al., 2002). This said, although first-episode depression is associated with amygdala enlargement, it was also reported that recurrent depression involves amygdala shrinkage (McEwen, 2004), possibly pointing to biphasic changes over the course of the illness.

\section{The functional outcomes of altered brain structure}

Reduced neurogenesis and impaired arborisation in the hippocampus, and altered neuronal structure in the PFC, led to a number of functional deficits. For example, patients with single and multiple past episodes of depression have poorer hippocampus-dependent memory recall (MacQueen et al., 2003). In animals subjected to chronic stressor treatments, spatial learning and spatial working memory in the Morris Water Maze was also impaired (Sousa et al., 
2000). Moreover, because excitatory hippocampal projections regulate the firing of cells in the ventral tegmental area, impaired hippocampal function could also contribute to anhedonia due to reduced dopamine transmission (Pittenger \& Duman, 2008). Additionally, because the hippocampus exerts negative-feedback control on the HPA axis and hence on glucocorticoid release, atrophy here could lead to disinhibition of CRH release from the hypothalamus, further exacerbating hyperactive stress responsivity (MacQueen et al., 2003). Like the hippocampus, the PFC might also contribute to negative feedback regulation of the HPA axis. For example, following 20 min of restraint, rats with lesions in the mPFC had significantly higher plasma levels of ACTH and corticosterone compared to sham-operated animals (Diorio et al., 1993). In addition to affecting later stress reactivity, chronic restraint stressors resulted in impaired attentional set-shifting performance in rats, and this effect was predicted by the decrease in dendritic arborisation in layer II/III pyramidal cells of the mPFC. Attentional set-shifting relies on mPFC function, so PFC remodelling may contribute to the attentional deficits seen in depression (Liston et al., 2006), and perhaps to the difficulty in shifting away from negative thoughts in depressed individuals.

At the same time, given the role of the amygdala in emotional memory, changes in this structure could contribute to anxiety and poor emotional control (Yu \& Chen, 2011). For example, reduced gray matter volume of, and functional coupling between, the amygdala and anterior cingulate may lead to over-activity of the amygdala and loss of emotional regulation (Pezewas et al., 2008). In a like fashion, depressed patients also show limbic, subcortical, and occipital overactivity as well as decreased activity in the MPFC and dorsolateral prefrontal cortex (dIPFC) in response to fearful facial expressions. This region-specific combination of hyper- and 
hypoactivity could reflect impaired cortical control of limbic regions leading to emotional dysregulation (Miskowiak et al., 2010).

\section{Antidepressant effects in the brain}

To somewhat limit the scope of this broad topic, depression is considered here to be a process involving a reduced plasticity and size of the prefrontal cortex and limbic brain regions. This owes at least in part to alterations in growth factor levels. Alterations in the size, plasticity and connectivity of these regions then likely lend to the behavioural features of depression. In contrast, antidepressants may exert their effects by enhancing the plasticity of the brain regions affected in depression.

Several classes of antidepressants increase BDNF in the major hippocampal subregions and in the PFC. Additionally, microinfusion of BDNF into the hippocampus has antidepressantlike effects (Yu \& Chen, 2011). In contrast, mice over-expressing a truncated BDNF receptor and heterozygous BDNF null mice (BDNF+/-) do not respond to antidepressant treatment in the forced swim test, indicating that this neurotrophin is necessary for antidepressant efficacy (Saarelainen et al., 2003). In this same study it was found that acute and chronic antidepressant treatment increased TrkB autophosphorylation, which is the first step in BDNF intracellular signaling (Saarelainen et al., 2003). TrkB signaling then leads to an increase in CREB phosphorylation, and this transcription factor promotes the expression of plasticity related molecules including BDNF (Yu \& Chen, 2011). At the same time, BDNF can activate CREB via the mitogen-activated protein (MAP) kinase cascade, creating a positive feedback loop. A positive feedback mechanism also seems to exist between serotonin signalling and BDNF. That is, CREB signalling is activated by serotonin acting at serotonin receptors, and CREB signalling 
leads to increased BDNF expression; BDNF then further promotes the function and development of serotonergic neurons (Pezawas et al., 2008).

In addition to BDNF, FGF-2 levels are also altered by antidepressant treatment. For example, repeated treatment with the antidepressants desipramine, fluoxetine, or mianserin all increased hippocampal protein and mRNA levels of FGF-2 in rats. Cortical levels were additionally elevated by chronic desipramine or mianserin treatment (Mallei et al., 2002). Furthermore, chronic treatment with fluoxetine plus olanzapine led to an increase in FGF-2 in the hippocampus, prefrontal cortex, and striatum of rats. Olanzapine is an atypical antipsychotic, and this particular combination of drugs can be effective against treatment resistant depression (Maragnoli et al., 2004). Human studies also demonstrate that depressed patients have lower levels of FGF-2 in the frontal cortex and CA4 hippocampal region, but treatment with selective serotonin reuptake inhibitors (SSRIs) attenuates this reduction in the frontal cortex (Gaughran et al., 2006). Microarray analysis also indicated that FGF-2 and its receptor were reduced in the anterior cingulate and dIPFC of depressed individuals, but SSRI treatment attenuated this decrease in the dIPFC (Evans et al., 2004). Hence, antidepressants may also exert some of their effects by elevating FGF-2. This is further supported by the observation that chronic peripheral administration of FGF-2 reduces anxiety-like behaviour in mice (Perez et al., 2009).

By promoting the proliferation and survival of neurons, these growth factors could presumably counter the structural and functional changes seen in depression. For example, antidepressant treatment can increase neurogenesis, as well as the number of synapses and spine density in the hippocampus (Pittenger \& Duman, 2008). Antidepressant treatment also reversed hippocampal atrophy in patients with post traumatic stress disorder (Pittenger \& Duman, 2008). 
If the same effect is true in depressed individuals, this could potentially improve many of the cognitive symptoms of the disease (Yu \& Chen, 2011). Furthermore, antidepressant treatment increases neuroplasticity, be it through an increase in neurotrophins or some other means. For example, fluoxetine treatment caused mature dentate granule cells to take on membrane properties of immature granule cells, in a more plastic state (Kobayashia et al., 2010). In other research, SSRIs enhanced long term potentiation (LTP) or prevented stress-induced impairments in LTP in the CA1 and hippocampal-PFC circuits (Pittenger \& Duman, 2008). Relating to this, there is some evidence for improved memory and cognition in humans following antidepressant treatment (Pittenger \& Duman, 2008).

\section{Erythropoietin}

Erythropoietin (EPO) is a cytokine that was originally known for its role in stimulating red blood cell production (Byts \& Sirén, 2009). It acts through the EPO receptor to activate Janus family tyrosine kinase 2 molecules. This then activates a number of other signaling pathways, including those involving signal transducers and activators of transcription (Stat molecules); phosphatidylinositol 3-kinase (PI3K)/Akt; Ras/extracellular signal regulated kinase (ERK1/2); nuclear factor kappa- B (NF-kB), and calcium (Byts \& Sirén, 2009). These pathways often lead to anti-apoptotic and proliferative effects.

EPO and its receptor have been detected in several brain regions including the hippocampus, internal capsule, cortex and midbrain. Conditions that limit oxygen supply and other stimuli which likewise activate hypoxia inducible factor cause increased expression of EPO. Such stimuli include hypoxia, ischemia, hypoglycemia, insulin, reactive oxygen species, and insulin-like growth factor (Byts \& Sirén, 2009). The neuroprotective effects of EPO are 
related to protection against DNA damage, reduced lipid peroxidation via an increased activity of cytosolic antioxidant enzymes, and the anti-inflammatory activity of EPO. EPO also stimulates angiogenesis and promotes vascular integrity. Furthermore, EPO is reported to stimulate neuronal differentiation and migration, neurite formation, and dendritic sprouting in addition to the activation of CREB and BDNF expression (Byts \& Sirén, 2009).

In light of these proliferative and neuroprotective effects of EPO, it is promising as a potential antidepressant and current research is investigating this possibility. For instance, rats treated with intraperitoneal injections of EPO $(500 \mathrm{U} / \mathrm{kg} \mathrm{bw} / \mathrm{d})$ on four consecutive days demonstrated antidepressant like effects in the forced swim and novelty induced hypophagia tests (Girgenti et al., 2009). Additionally, BDNF mRNA in the dentate gyrus was upregulated as a result of EPO treatment (Girgenti et al., 2009). Further, after intracerebroventricular infusions of EPO, hippocampal mRNA levels of BDNF, VGF and neuritin were upregulated, and mRNA levels of tumor necrosis factor receptor associated death domain (TRADD) were reduced. The down-regulation of TRADD may play a role in the anti-apoptotic effects of EPO, while VGF and neuritin may have their own antidepressant effects (Girgenti et al., 2009; Son et al., 2012). VGF, BDNF, and neuritin are likewise all increased by exercise (Girgenti et al., 2009) and EPO may mediate this effect since exercise would increase oxygen demand leading to an increase in EPO expression as mentioned above. Exercise is known to have antidepressant effects, yet in patients who are severely depressed, exercising may require more motivation than they could possibly gather. In this case administering a drug form of EPO may be an attractive alternative.

Other animal studies likewise pointed to the antidepressant potential of EPO. In mice, intraperitoneal injections of EPO at a dose of $5,000 \mathrm{U} / \mathrm{kg}$, three times per week for two weeks, 
had anxiolytic and antidepressant-like effects in the forced swim, open field, elevated plus maze and novel object test, while also promoting hippocampal neurogenesis (Osborn et al., 2013). Moreover, daily intraperitoneal injections of EPO for one week led to increased neurogenesis in the subgranular zone of the hippocampus in vivo (Hoon et al., 2012). In vitro, EPO increased neuronal differentiation of hippocampal progenitor cells, as indicated by BrdU/MAP2 colabelling. Furthermore, EPO and its carbamylated derivative, CEPO, enhanced the spine density and dendritic length of neural progenitors. CEPO is a noteworthy therapeutic alternative to EPO as it does not have haematopoietic effects, while it still shows neuroprotective activity (Hoon et al., 2012). Finally, in stroke research, four days of EPO injections (5,000 U/kg bw, IP) also increased protein levels of FGF-2 in ischemic cortical tissue (Koegh et al., 2007), indicating the potential for EPO to positively affect this growth factor in the context of depression.

A number of human studies also indicated antidepressant effects of EPO. For example, when healthy subjects were given a single injection of EPO, they showed a reduced occipitoparietal response to, and reduced behavioural recognition of, fearful facial expressions seven days after the injection (Miskowiak et al., 2007). This is similar to the effects of other established antidepressants and opposite to what is seen in depression (Miskowiak et al., 2007). Similarly, a one-time EPO injection in depressed patients led to reduced hippocampal, ventromedial prefrontal and parietal cortical responses during the encoding of negative pictures compared to positive pictures. This effect may counteract the negative memory bias seen in depression (Miskowiak et al., 2009). There was also a decrease in left amygdala-hippocampal and parietal response to fearful faces in depressed individuals three days after EPO treatment, which again is similar to the effects seen with conventional antidepressants (Miskowiak et al., 2010). Based on 
this evidence, and consistent with a neuroplastic theory of depression, there is reason to believe that EPO could be a useful antidepressant.

The intent of this research was to examine the effects of peripherally administered EPO on depressive-like behaviours and hippocampal growth factors in an animal model of depression. It was predicted that the depressive-like behaviours, comprising social avoidance and anhedonia, would be induced by a chronic stressor, but that these behaviours would be reversed or reduced with EPO treatment. Furthermore, it was predicted that a chronic stressor would reduce hippocampal levels of BDNF, but that EPO would normalize this outcome. It was also expected that a chronic stressors might elevate levels of BDNF mRNA in the PFC and amygdala, and that EPO might also increase BDNF expression in these brain regions. Chronic stressors may lead to elevated hippocampal levels of FGF-2 in a protective manner, and a reduction in FGF-2 mRNA levels in the PFC, and it was expected that the level of FGF-2 would be elevated by EPO treatment in each of these brain regions. Such results would support the development of EPO as a neuroplasticity-enhancing antidepressant. 


\section{Chapter 2: Materials and Methods}

\section{Animals}

Male Long Evans rats (Charles River Laboratories, St. Constant, QC, Canada) initially weighing $250 \mathrm{~g}$ were singly housed in $45 \times 24 \times 20 \mathrm{~cm}$ polypropylene cages. Rats were given 5 days to acclimatize to their surroundings in a controlled environment with temperature $\left(21^{\circ} \mathrm{C}\right)$ kept constant, a 12-h light-dark cycle with lights on from 0800 to 2000 hours, and free access to food. Animals were also given free access to water, apart from the hour prior to the sucrose consumption test, during which time water was unavailable. The experimental procedures were approved by the Carleton University Animal Care Committee and met the guidelines set out by the Canadian Council on Animal Care.

\section{Procedure}

All animals were initially handled daily at 8am over a 6-day period and general disposition was noted. During this time sucrose consumption training was carried out on 6 consecutive days during this baseline period. The details of this procedure are described below. At the end of these six days, animals were randomly assigned to treatment groups so that each group was matched for baseline sucrose consumption and general disposition, i.e. each group contained approximately the same number of animals noted to be especially nervous upon handling. Rats were exposed to either chronic unpredictable stressors or no stressors. These groups were further subdivided so that animals in each condition received either saline or EPO injections ( $\mathrm{n}=8$ /group).

Following the six-day baseline period, the 21-day chronic mild stressor exposure began. This entailed exposing half of the animals to twice daily physical stressors. The morning stressor occurred between 8 am and $11 \mathrm{am}$, and the afternoon stressor occurred between noon and $4 \mathrm{pm}$. 
Stressors used were: $15 \mathrm{~min}$ restraint in a plastic baggie; $30 \mathrm{~min}$ intermittent startle noise directed at the animals' cages; five min tail pinch; one hour exposure to dirty bedding from unfamiliar animals; one hour of wet bedding; 36 hours of light. All animals received the same stressor at a given time, and stressors were applied in a random order to minimize their predictability. For all animals, the final stressor received was the bag restraint. Stressed and nonstressed rats were housed in separate rooms to avoid transmission of stress pheromones, and different lab coats were used in each room.

Throughout the stressor exposure period, animals were also observed 2 to 3 times weekly and scored for overt signs of stress. Each animal was scored as either a zero or 1 for displaying piloerection, ptosis, cowering or audible squeals. For each animal, the daily average score (out of 4) from the final week of the study was used to analyze potential correlations between overt signs of stress and other observations.

\section{EPO treatment}

On the 11th, 14th, 17th, and 20th day following stressor initiation, half of the stressed animals and half of the non-stressed controls received intraperitoneal injections of erythropoietin (Millipore, Etobicoke) at a dose of 5,000 U/kg in $0.4 \mathrm{~mL}$ saline. The other half of these animals received saline injections $(0.4 \mathrm{~mL}$ of $0.9 \% \mathrm{NaCl})$ at the same time points. Injections were administered at approximately noon each day.

\section{Sucrose consumption test}

During the six-day baseline period animals had 23 hours of daily access to a bottle of pure water and a bottle of a sucrose solution. These were made using tap water to match the liquid consumed during non-test days. Solutions were prepared on the afternoon prior to the test 
and stored in home rooms overnight to allow water volumes to adjust to the humidity of the home room. The cage position of the bottle containing the sucrose solution alternated from left to right each day to avoid the development of a conditioned place preference. Labelled bottles were weighed at the start and end of each 23-hour consumption period and the difference was calculated to estimate the volume of liquid consumed. The density of the sucrose solution was accounted for when calculating the volume of sucrose solution consumed. For the first two days of the sucrose consumption test baseline, bottles contained a $2 \%$ sucrose solution. Thereafter the solution was $1 \%$ for the duration of the study. The initially higher concentration of $2 \%$ was used to counteract potential neophobia. After the initial six-day baseline period, the sucrose consumption test was conducted twice weekly, with animals having access to a $1 \%$ sucrose solution and pure water for 23 hours on two consecutive days. 23 -hour periods spanned from 9am until 8am the next day. Between 8am and 9am bottles were weighed and animals were handled and their overt behaviour scored.

\section{Social approach testing}

To assess social avoidance as a potential depressive-like feature in stressed animals, a three-chamber social approach test was used, as previously described (van der Kooij et al., 2013). In this test, animals were placed into a three-chambered apparatus where the test subject could either spend time in the empty centre chamber, or enter and explore the two side chambers. Each chamber of the three-chamber apparatus was composed of a polypropylene bin $(60 \mathrm{x} 38 \mathrm{x}$ $29 \mathrm{~cm}$ ) and three of these chambers were connected in series. During the test period, each side chamber contained either an enclosed basket containing a novel object, or an enclosed basket containing an unfamiliar conspecific as a social target. The enclosed baskets had regularly spaced holes to allow visual and olfactory contact with their contents. The side chamber 
containing the social target was alternated on each trial. Animals used as social targets were acclimated to the enclosed baskets for $10 \mathrm{~min}$ per day for four days prior to the start of testing. 24 hours after their final stressor, test animals received a 10-min acclimation period in the empty test chamber, followed immediately by the 10-min social approach test (with the social target and novel object present). The acclimation and test periods were video-taped and later scored blindly. For each session, the time spent in the right and left chamber was measured. During the acclimation period, the number of chamber crossings was counted to look for general locomotor effects. For the social approach test period, the time spent in contact with the social target and novel object was also measured.

The social approach test was completed between $8 \mathrm{am}$ and noon each day to limit the effect of variations in circadian rhythm on animal behaviour. Additionally, a subset of animals from each treatment group was tested each day. This was done to limit the effects of any potential changes in test conditions between test days. All test animals were handled daily throughout experimentation.

\section{Tissue collection}

Brain removal and tissue collection followed the procedure previously described (Audet et al., 2011). 24 hours after social approach testing, rats were sacrificed by rapid decapitation. Brains were immediately removed and placed on a stainless steel rat brain matrix positioned on a block of ice. The matrix had a series of slots that guided razor blades to provide coronal brain sections. Once the brains were sliced, tissue punches were collected from the PFC, hippocampus, and amygdala. Punches were immediately placed in tubes resting on dry ice and then stored at $-80^{\circ} \mathrm{C}$ for subsequent determination of growth factor mRNA expression. 


\section{Reverse transcription-quantitative polymerase chain reaction analysis (RT-qPCR)}

As described previously (Audet et al., 2011), trizol was used to homogenize brain tissue and total RNA was isolated according to the manufacturer's protocol (Invitrogen, Burlington, ON, Canada). Total RNA was reversed transcribed with Superscript II reverse transcriptase (Invitrogen, Burlington, ON, Canada). Aliquots of the cDNA were analyzed in simultaneous quantitative polymerase chain reactions employing SYBR green detection according to the manufacturer's protocol (Stratagene Brilliant qPCR kit). Data was collected using a Stratagene MX-4000 real time thermocycler. All primer pairs generated amplicons between 80 and 120 base pairs. MX-4000 software calculated primer efficiency using the slope relation between absolute copy number and cycle threshold. All primer pairs had a minimum of $90 \%$ efficiency. Primers for GAPDH and synaptophysin were used as reference genes. To normalize the expression of genes of interest, average cycle threshold $\left(\mathrm{C}_{t}\right)$ of the reference genes was

subtracted from the gene of interest $C_{t}\left(\Delta C_{t}\right)$. The $2^{-\Delta \Delta C t}$ method (Livak \& Schmittgen, 2001; Schmittgen \& Livak, 2008) was used to convert $\Delta \mathrm{C}_{\mathrm{t}}$ values to mRNA fold changes relative to the no stressor-saline control group (calibrator). Primer sequences used for qPCR were as follows: Rat GAPDH, forward: GCCATCAACGACCCCTTCAT, reverse:

CCGCCTGCTTCACCACCTTC; Rat synaptophysin, forward:

CTATGATGGACTTTCTGGCTAC, reverse: TAATGTTCTCTGGGTCCGTG; Rat BDNF, forward: GGACATATCCATGACCAGAAAGAAA, reverse: GCAACAAACCACAACATTATCGAG; Rat FGF-2, forward: GACCCACACGTCAAACTAC, reverse: CGTCCATCTTCCTTCATAGC. 


\section{Statistical analyses}

Data was analyzed using the Statistical Package for Social Sciences (SPSS) version 22. A 2 (unstressed or stressed) x 2 (saline or EPO) between-groups analysis of variance was used to compare group means for measures in the social approach test, namely: the total number of chamber crossings during acclimation, the total time spent in contact with the novel object or social target, the latency to interact with the social target and novel object, and the number of entries into each side chamber. Sociability was also assessed in each treatment group by comparing the total time spent in the chamber containing the social target versus the novel object. In this case, a $2 \times 2 \times 2$ mixed ANOVA was used, with the presence of the object versus social target as the within subjects factor. A $2 \times 2$ repeated measures ANOVA was used to examine the effect of time in the sucrose consumption test. Sucrose preference was considered to be the percent of the total liquid intake that was sucrose solution. The significance level was set at $\mathrm{p} \leq 0.05$ for all statistical comparisons. T-tests were used in follow-up comparisons, with a Bonferonni correction to maintain an alpha value of 0.05. Finally, Pearson's correlational coefficients were calculated to examine possible correlations between behavioural data and growth factor levels. 


\section{Chapter 3: Results}

\section{Sucrose consumption test}

In the sucrose consumption test, sucrose preference did not vary as a function of time, drug treatment, or stressor condition (Figure 1). Additionally, there was no significant interaction between any of the variables $(p>0.05)$. Tests of between subjects effects indicated that no interaction was present between drug and stressor condition, nor were significant main effects present for these factors $(p>0.05)$. Tests of within subjects effects likewise indicated there was no significant effect of time on sucrose preference nor were interactions present $(\mathrm{p}>0.05)$. During the baseline period, the average preference for sucrose solution varied from $8.5 \%$ to $93.6 \%$ with an average preference of $67.4 \%$ and a standard deviation of $31.9 \%$. 


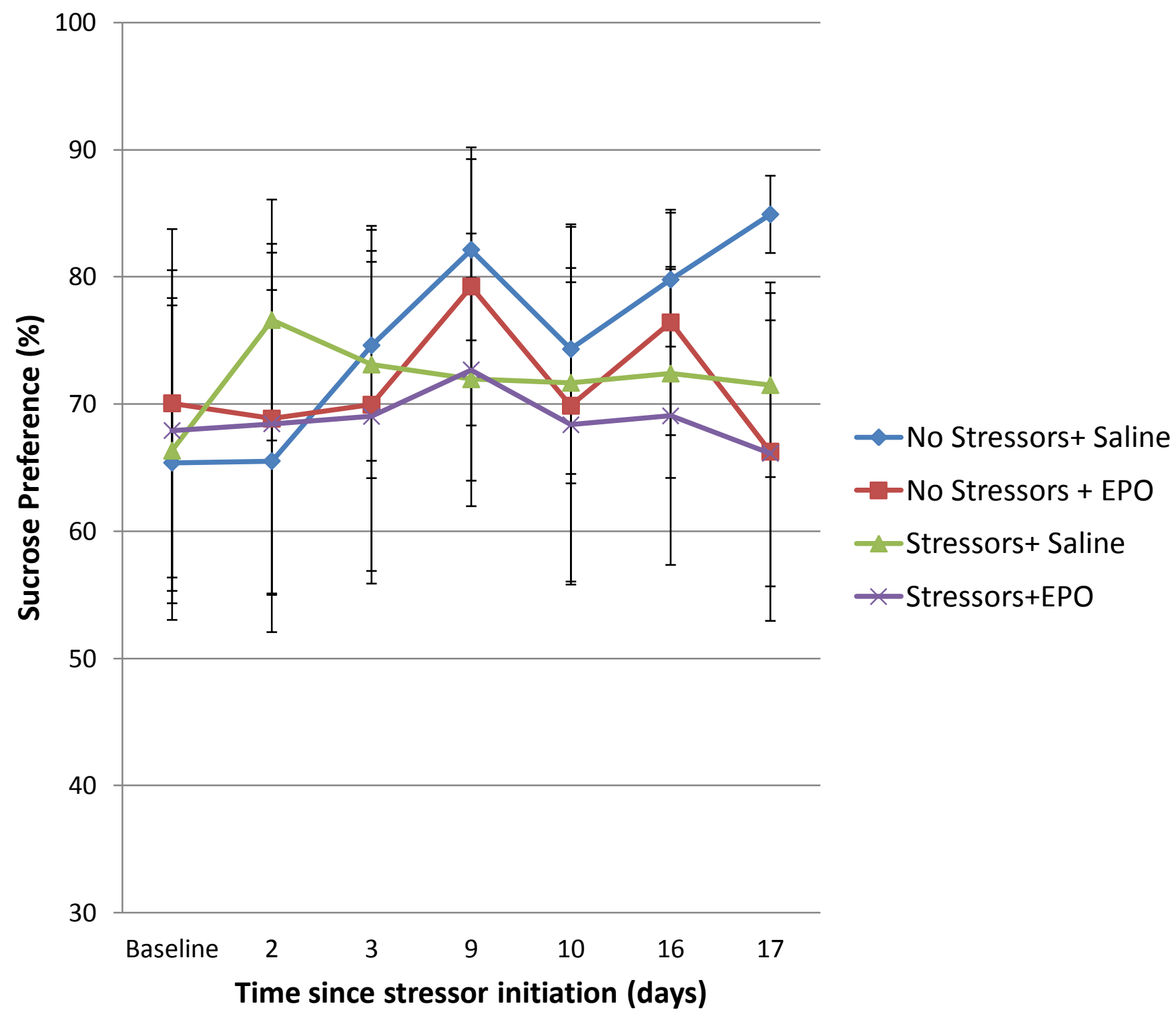

Figure 1: Results of the sucrose consumption test.

Sucrose and plain water were made available to rats for 23-hour periods during a 6-day baseline training period and twice per week after stressor initiation. Sucrose preference was defined as the percent of total liquid intake that was sucrose solution. Rats ( $\mathrm{n}=8 /$ group) were treated with 21 days of chronic unpredictable stress (Stressors) or handled daily (No Stressors), in addition to receiving injections (EPO or saline) every 3 days for 2 weeks. Values are expressed as means \pm S.E.M. 
Additionally, upon visual inspection, there was no clear trend in the change in percent preference amongst individual animals in a given treatment group (Figure 2).

A



C

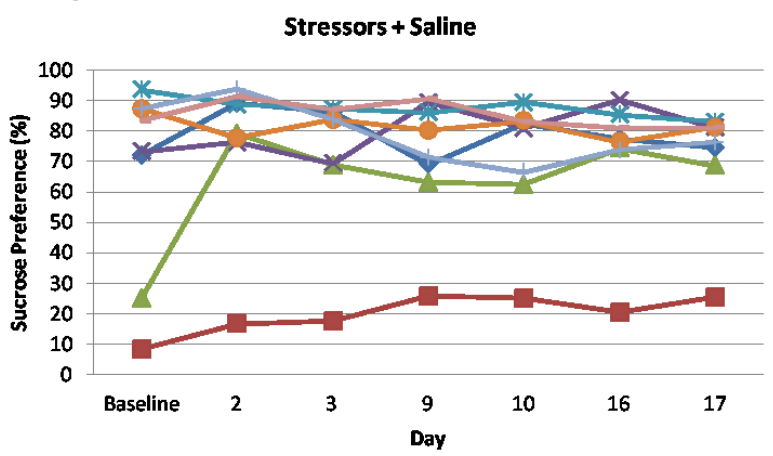

B

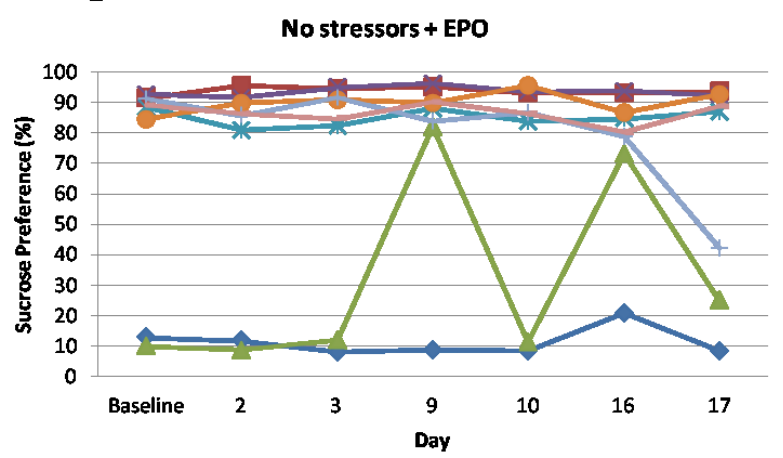

D

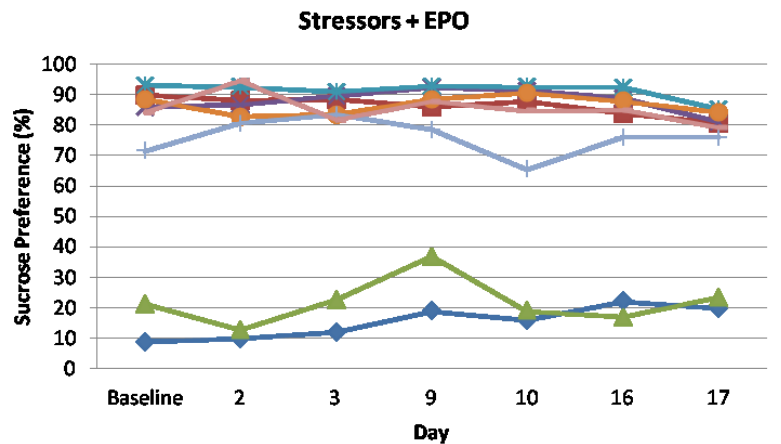

Figure 2: Preference for a 1\% sucrose solution in individual animals. Preference for a sucrose solution was defined as the percentage of liquid intake that was consumed as a sucrose solution when animals had access to pure and sugar water during 23-hour measurement periods. Results are presented by treatment group, with each line representing the preference in an individual animal. 


\section{Social approach testing}

General locomotion, reflected by chamber crossings during the acclimation period (Figure 3A), varied as a function of the interaction between the Drug and Stressor treatments, $\mathrm{F}(1,28)=4.263, \mathrm{p}=0.048$. Follow-up comparisons indicated that interaction effects were just shy of significance. In stressor-treated animals there was a trend towards reduced locomotion following EPO treatment compared to saline treatment $(\mathrm{p}=0.07)$. Additionally, amongst salinetreated animals, stressor exposure tended to increase locomotion $(\mathrm{p}=0.07)$.

The latency of test subjects to interact with a social target (Figure 3B) did not vary as a function of the stressor condition, the drug treatment, or in relation to the interaction between these variables, $\mathrm{F}$ values $<1$. Although the effects of the EPO treatment on the latency to interact with a social target did not reach statistical significance, $F(1,28)=3.235, \mathrm{p}=0.083$, it did appear that compared to EPO-treated animals, saline-treated rats interacted with social targets somewhat more readily $(\mathrm{M} \pm \mathrm{SEM}=32.94 \pm 7.50 \mathrm{~s}$ and $57.94 \pm 11.77 \mathrm{~s}$ for saline and $\mathrm{EPO}$ treated animals, respectively).

Time spent in direct interaction with an unfamiliar conspecific (Figure 3C) varied as a function of stressor condition $\mathrm{F}(1,28)=5.505, \mathrm{p}=0.026$, where stressor-exposed animals spent less time interacting with the social target $(245.56 \pm 29.54 \mathrm{~s})$ compared to non-stressor exposed animals (326.44 $\pm 18.05 \mathrm{~s})$. There was no significant main effect of drug, nor was the Stress $\mathrm{x}$ Drug interaction significant, $F$ values $<1$. Sociability in an animal can also be defined as spending significantly more time in the chamber containing the social target compared to the chamber containing the novel object (Kaidanovich-Beilin et al., 2011). By this definition, a 2 x 2 x 2 mixed ANOVA indicated the presence of sociability in all treatment groups, as a significant main effect of the target versus object presence existed $F(1,28)=55.705, p=0.000$ (Figure 3D). 
The time spent interacting with a novel object (Figure 3E) did not significantly differ based on stressor condition, drug treatment, or an interaction between these factors. However, the effect of stressor condition neared significance $F(1,28)=3.288, p=0.081$. The stressor-exposed and non-stressor-exposed rats spent an average of $117.00 \pm 15.25 \mathrm{~s}$ and $84.94 \pm 9.89 \mathrm{~s}$ interacting with the novel object, respectively.

The number of entries into the chamber containing a social target or novel object also did not differ significantly based on drug treatment, stressor condition, or an interaction between these factors, F-values $<1.3$. 




B

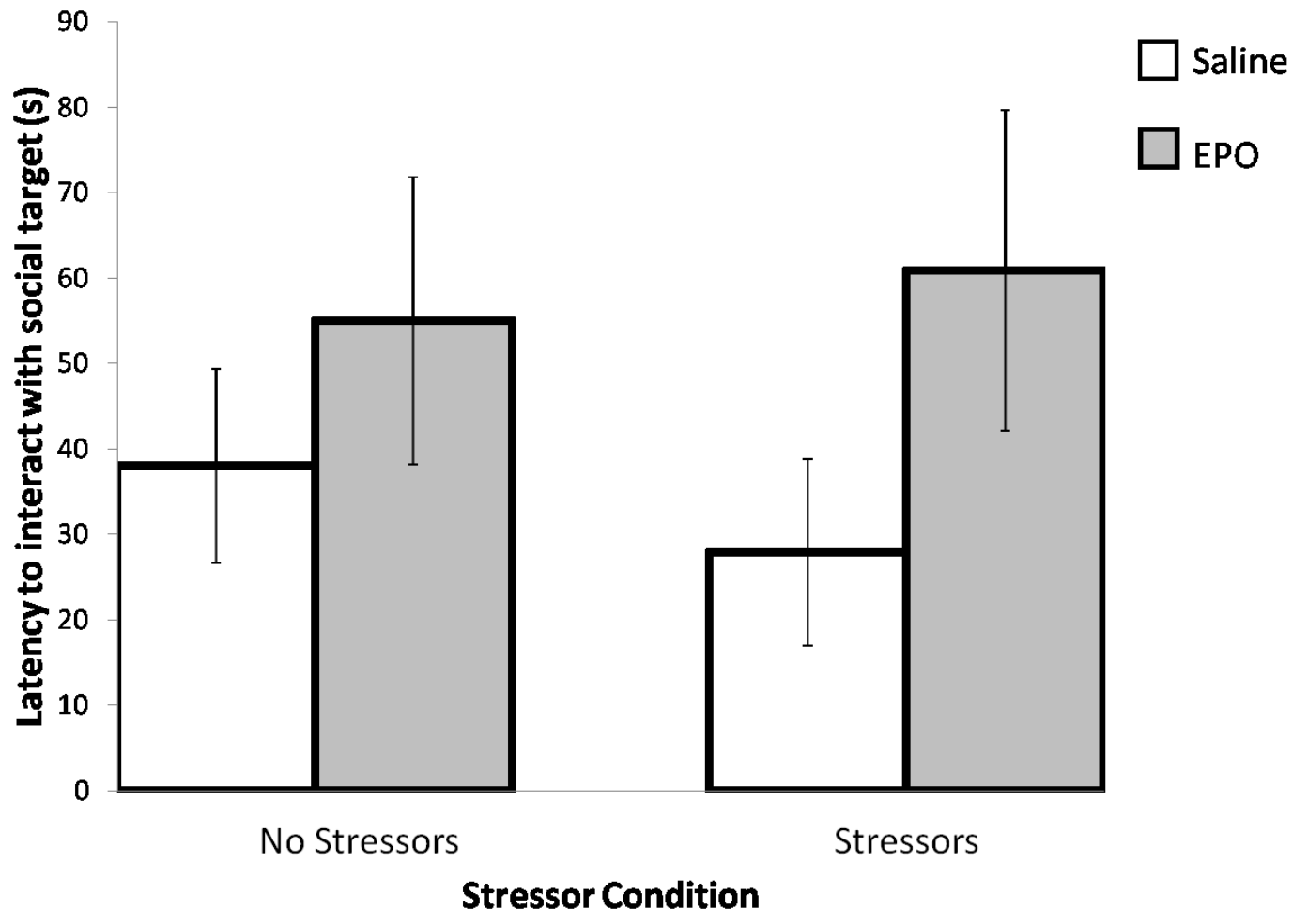


C

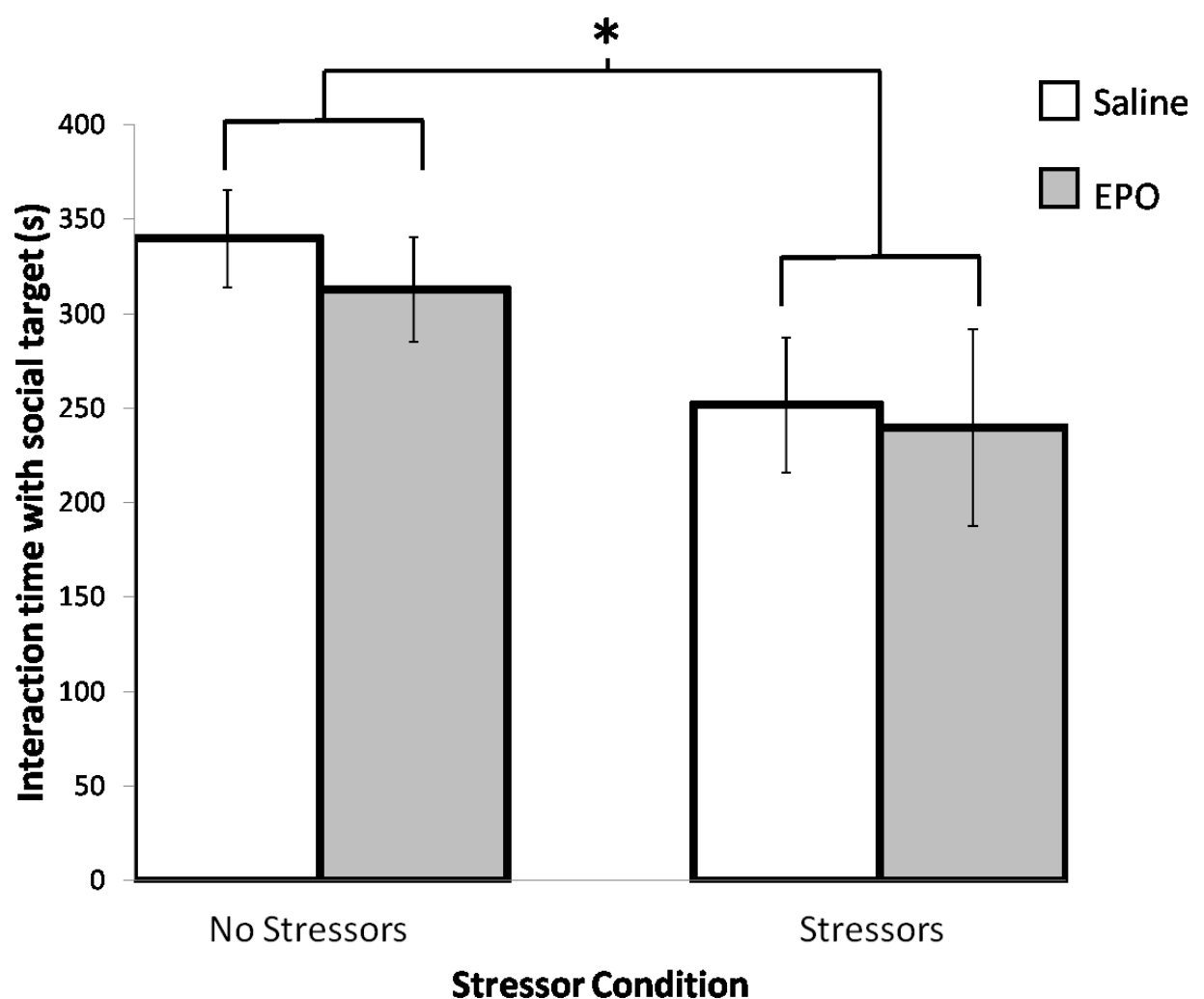

D




$\mathbf{E}$

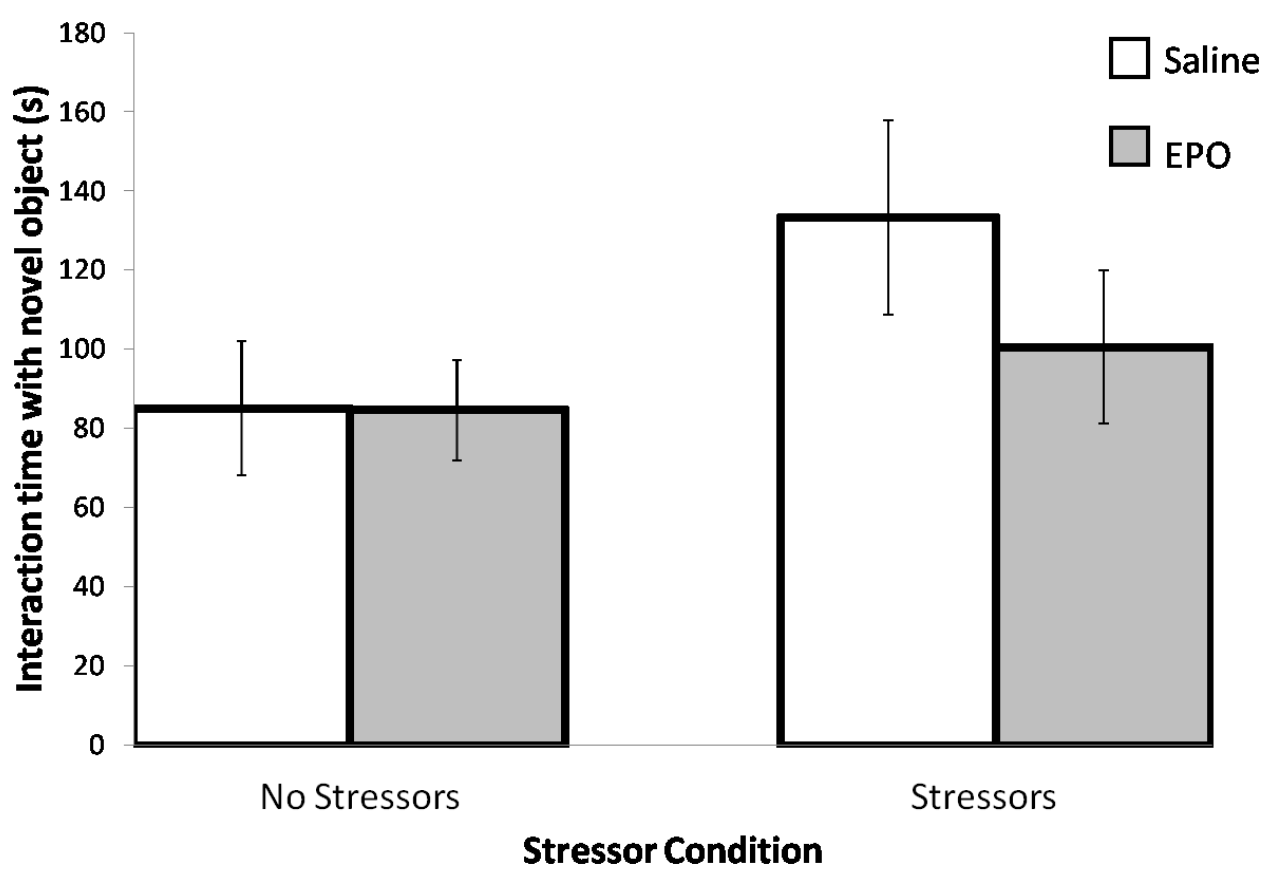

Figure 3: Results of social approach testing.

Rats ( $\mathrm{n}=8$ /group) were treated with 21 days of chronic unpredictable stress (Stressors) or handled daily (No Stressors), in addition to receiving injections (EPO or saline) every 3 days for 2 weeks. In a three-chambered social approach test, results are reported for a) the number of chamber crossings made during the acclimation period; b) the latency to interact with an unfamiliar social target; c) the test subjects' time spent in interaction with an unfamiliar conspecific; d) the relative time spent in the social target versus novel object side chambers; and e) the test subjects' time spent in interaction with an unfamiliar object. Values are expressed as means \pm S.E.M. $* p<0.05$, $* * \mathrm{p}<0.01$. 


\section{Growth factor mRNA expression}

In the prefrontal cortex, results of mRNA analysis indicated that BDNF mRNA expression was significantly reduced by both the stressor and EPO treatments (Figure 4A), $F(1,28)=4.731, p=0.0382$ for stressor condition; $F(1,28)=39.620, p<0.0001$ for EPO. In contrast, FGF-2 mRNA expression was not significantly impacted by experimental treatments, F-values $<0.3$.

In the hippocampus, BDNF mRNA expression was not significantly altered by stressor treatment, $\mathrm{F}(1,28)=1.488, \mathrm{p}=0.2331$, nor was $\mathrm{BDNF}$ expression significantly affected by EPO administration, although there was a non-significant increase in BDNF mRNA levels in this case (Figure 4B), $\mathrm{F}(1,28)=3.872, \mathrm{p}=0.0594$. Again, FGF-2 mRNA expression was not affected by either treatment, F-values $<0.3$.

In the amygdala, BDNF mRNA expression was not altered by stressor treatment. However, EPO treatment led to a significant increase in BDNF expression in this brain region (Figure 4C), $F(1,28)=9.627, p=0.0043$. Stressor exposure and EPO treatment interacted to affect FGF-2 mRNA expression in the amygdala (Figure 4D), $F(1,28)=6.401, \mathrm{p}=0.0173$. Follow-up comparisons indicated that with stressor exposure, EPO significantly elevated FGF-2 expression, $\mathrm{p}=0.025$. Furthermore, without drug treatment, stressor exposure significantly reduced FGF-2 expression, $\mathrm{p}=0.032$. Unfortunately mRNA samples from this brain region had unacceptably low purity, with a 260/230 ratio of absorbances well below 2.0 (Thermo Scientific, n.d.). Therefore, results for this brain region must be considered with caution. 
A

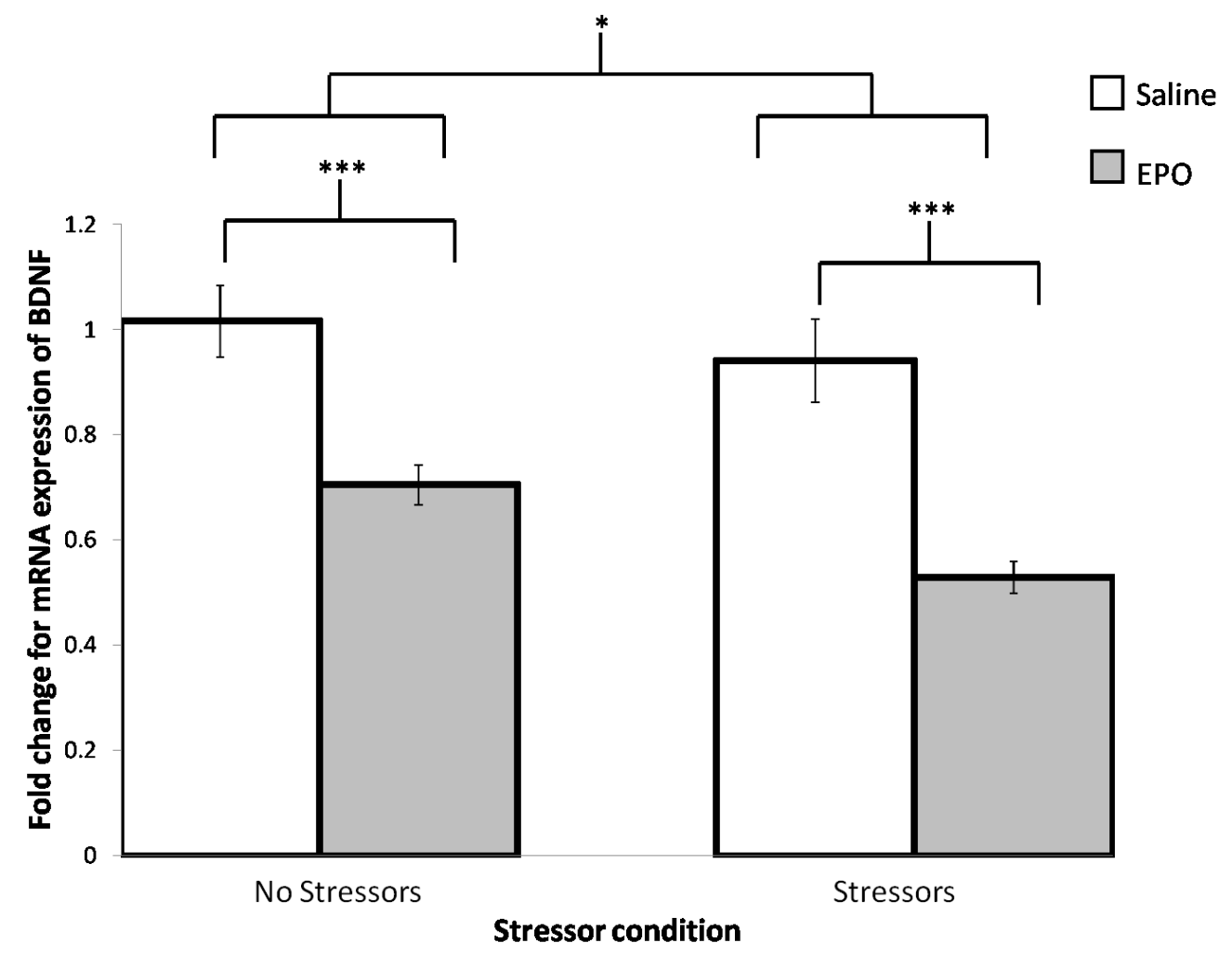

B

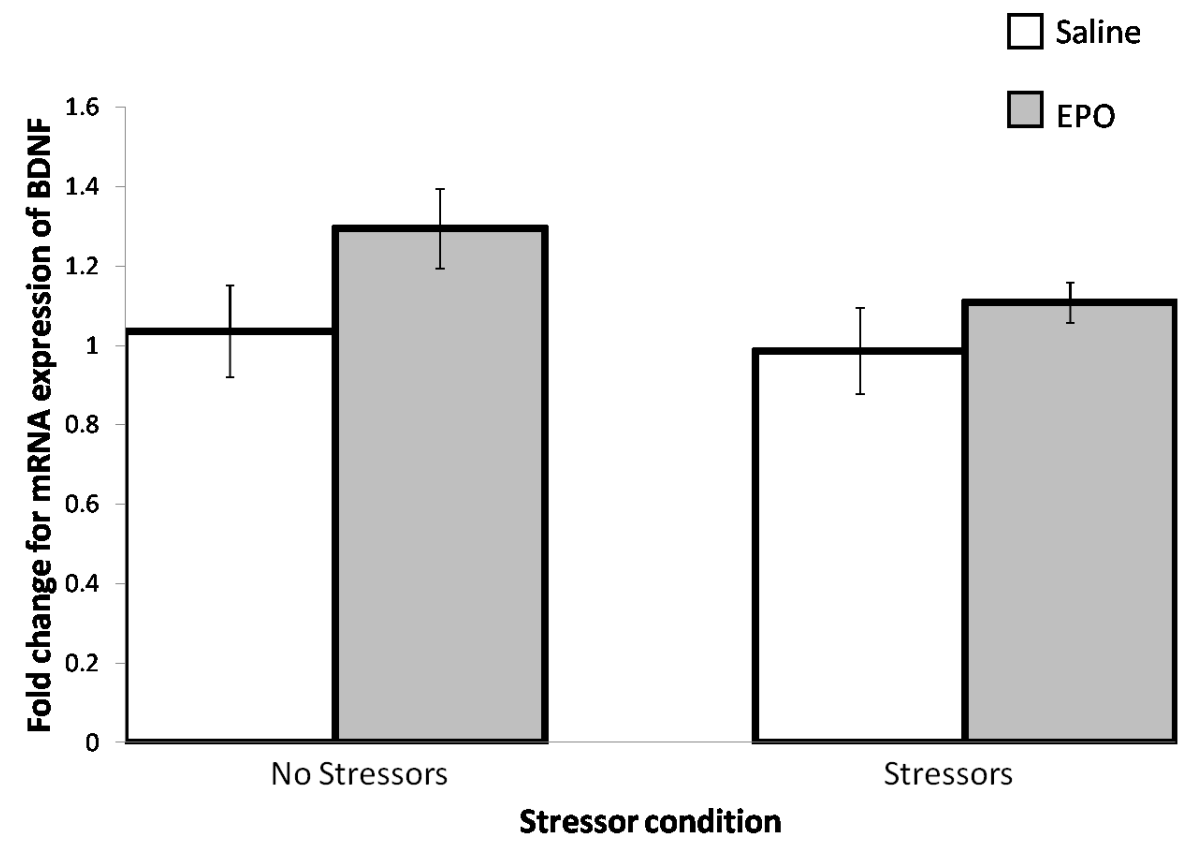


C

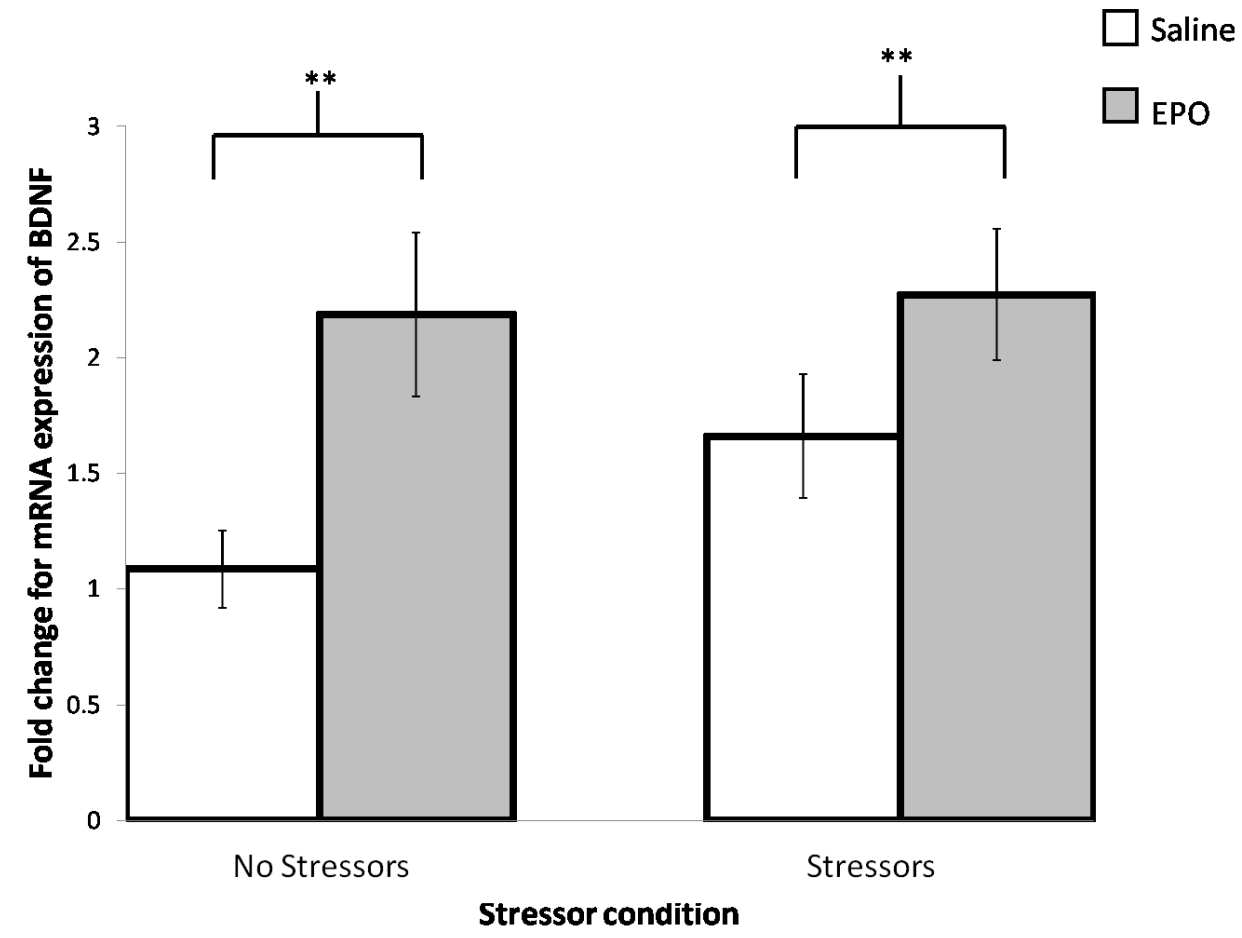

D

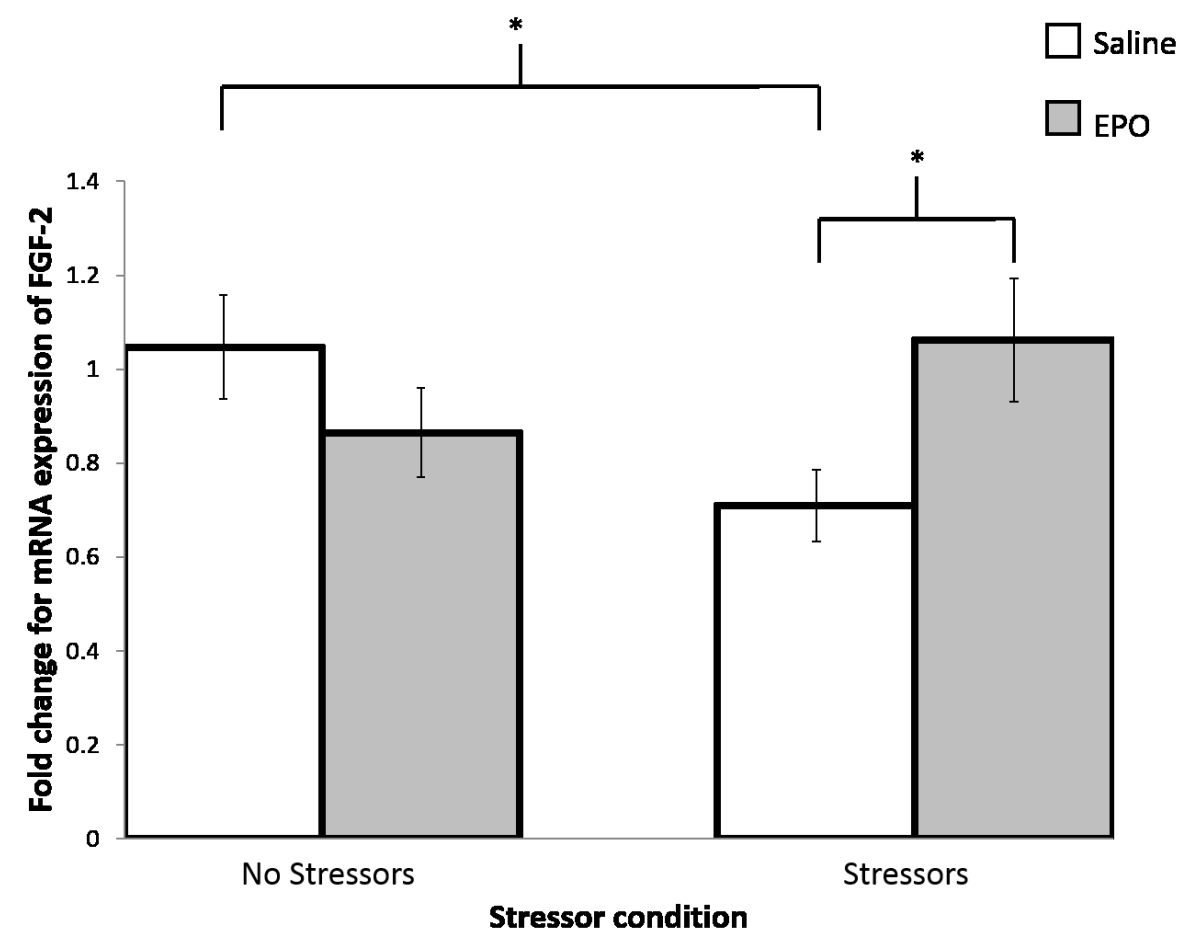


Figure 4: Results of BDNF and FGF-2 mRNA analysis

At the end of the three-week stressor period, animals underwent behavioural testing followed by rapid decapitation so that tissue was collected 2 days after the final stressor was administered. Graphs represent the mRNA analysis of A) BDNF in the prefrontal cortex, B) BDNF in the hippocampus, C) BDNF in the amygdala, and D) FGF-2 in the amygdala. Values are expressed as means \pm S.E.M. ${ }^{*} \mathrm{p}<0.05,{ }^{*} \mathrm{p}<0.01,{ }^{*} * \mathrm{p}<0.0001$.

\section{Correlations}

Amongst animals treated with stressors and saline, some correlations were found. Specifically, the change in sucrose preference (final preference score minus the baseline preference score) correlated positively with BDNF level in the hippocampus, $\rho=0.794, p=0.033$, with FGF-2 in the hippocampus, $\rho=0.881, p=0.009$, and with BDNF in the amygdala, $\rho=0.775$, $\mathrm{p}=0.024$. In this treatment group, interaction time with a social target also correlated positively with BDNF level in the hippocampus, $\rho=0.778, p=0.039$, and with BDNF in the amygdala, $\rho=0.804, p=0.016$. However, despite these trends, consistent correlations between behavioural and biochemical data were not consistently found in other treatment groups. 


\section{Discussion}

In this experiment we investigated the antidepressant-like properties of EPO, using chronic mild stressor exposure to induce depressive-like symptoms in rats. Depressive-like behaviours were measured in the sucrose consumption test and a 3-chamber social approach test, and mRNA levels of BDNF and FGF-2 were additionally measured in the prefrontal cortex, hippocampus and amygdala. It was hypothesized that chronic mild stressors would induce the depressed-like behaviours of anhedonia and social avoidance in the sucrose consumption and social approach test, respectively, but that EPO might reverse these depressed-like behavioural effects. Additionally, it was expected that specific alterations in growth factors would occur, but as will be discussed, the initial hypotheses did not prove to be correct.

\section{Anhedonia in the sucrose consumption test}

A reduction in reward system activity is theorized to occur in depression, and anhedonia, or an inability to experience pleasure, is a core feature of the disease (Moreau, 2002). Therefore, demonstrating that chronic mild stress results in reduced responsiveness to rewarding stimuli is important in animal models of depression (Moreau, 2002). Reduced intake of a palatable solution is said to reflect anhedonia in animal models of depression; however, a reduced preference for sucrose solutions following stressor treatment is not always observed (Grønli et al., 2005).

In the present study, no effect was seen as a result of stressor or EPO treatment in the sucrose consumption test. A high degree of variability in the animals' innate preference for sucrose solution was apparent, making it difficult to detect any significant change in the average preference between treatment groups. 
Given the clear lack of response in the sucrose consumption test in this study, it is worth looking to other literature for possible explanations for the lack of effect, or to improve the methods used here. In a number of studies that successfully demonstrated stressor-induced 'anhedonia', one common difference was the inclusion of a food and water deprivation period prior to the initiation of the sucrose consumption test (Bessa et al., 2013; Grønli et al., 2005; Papp et al., 1991; Willner et al., 1987). Deprivation periods ranged from 4 to 23 hours and may serve to stimulate liquid consumption once bottles are re-introduced to the cage (Konkle et al., 2003). However, the consumption test period in these studies was most often limited to 1 hour. Given that our test period was 23 hours, stimulating consumption was unnecessary as there was less of a time constraint to measure an appreciable liquid intake. The 23 -hour test period without a prior water deprivation period may also be more representative of the animal's daily preference for sugar or plain water, while avoiding potential effects of individual variations in daily drinking patterns when shorter measurement periods are used (Strekalova et al., 2011). Excluding a deprivation period prior to the start of the test might also avoid complications of metabolic effects (Konkle et al., 2003). Still, for the purpose of demonstrating reduced sucrose consumption or preference following stressor treatment, a food and water deprivation period prior to a shorter measurement period may be more effective. For example, some argue that this method may reduce within-group variability and increase differences between groups, facilitating detection of differences in sucrose consumption between groups (Muscat \& Willner, 1992).

Another common feature of some studies was the inclusion of food and water deprivation as a stressor (Bessa et al., 2013; Grønli et al., 2005; Muscat \& Willner, 1992; Papp et al., 1991; Willner et al., 1987). Given that the stressors included food deprivation, it was suggested that 
reduced body weight and reduced sucrose consumption could be due to the metabolic effects of food deprivation, and that reduced sucrose consumption in stressor-exposed animals could be attributed not only to hedonic changes, but also to changes in body weight and hunger (Forbes et al., 1996). When a modified stressor regimen was used which excluded food and water restriction, animals given the modified stressors did not significantly differ from controls in their body weight, total sucrose consumption or sucrose preference (Forbes et al., 1996). Elsewhere however, changes in sucrose consumption have been observed relative to the animals' body weight (Grønli et al., 2005) and also in studies that did not use food and water deprivation as a stressor (Willner, 1997), indicating that stressor-induced anhedonia should be demonstrable without using food and water deprivation as a stressor.

Stressor severity is another factor to consider when comparing our methods to other papers. Many studies used more frequent and longer-lasting stressors than what was done here, including 5 or more stressors per day, and stressor exposure during the day and night (Bekris et al., 2005; Willner et al., 1987). More frequent and varied stressors had a greater effect in reducing sucrose intake, and night-only stressors (during the animals' active phase) were less reliable in inducing anhedonia-like symptoms (Muscat \& Willner, 1992). However, a simple stressor schedule of social pairing, cage wetting, and a cage tilt, each applied twice weekly, was as effective as much more severe stressor paradigms, so it is unlikely that stressor severity was a problem in our experiment.

The strain of animal may also be an important factor when conducting the sucrose consumption test. A commonly used strain in successful studies was the Lister hooded rat (Cheeta et al., 1994; Muscat \& Willner, 1992; Papp et al., 1991; Willner et al., 1987) although 
results using this strain were not always successful in demonstrating stressor-induced anhedonia (Forbes et al., 1996). A reduction in sucrose intake and preference as a result of stressor exposure was also demonstrated in Sprague-Dawley and Wistar rats (Bekris et al., 2005). In studies using Long Evans rats, as used here, sucrose consumption was reduced following stressor exposure (Baker et al., 2006; Valverde et al., 1997), but not always (Konkle et al., 2003). Sucrose preference, or sucrose consumption relative to total liquid intake, was likewise not always affected in Long Evans rats, even with the inclusion of a food deprivation period prior to testing, and stressors that included food and water deprivation and over-night stressors (Baker et al., 2006; Konkle et al., 2003).

As a point in favour of the methods used here, singly-housed, stressor-exposed animals displayed differences in sucrose intake earlier and with greater significance, compared to stressor-exposed animals housed in pairs (Muscat \& Willner, 1992). In the same study, singly and pair-housed animals did not differ in sucrose consumption when no stressors were applied (Muscat \& Willner, 1992). Additionally, a 1\% sucrose concentration is ideal for detecting differences in reward value based on total consumption. At higher concentrations, increasing concentrations may be preferred, but total intake is reduced (Muscat \& Willner, 1992). Other factors consistent with many studies already mentioned included the time frame allowed for stressor effects to become evident (2-3 weeks); regularly alternating the side of the cage which contained a sucrose solution; including a training period where animals could get used to the sucrose solution; the number of animals used (10 was most common); and matching the animal composition of treatment groups based on baseline sucrose consumption. 


\section{Social avoidance in a three-chambered social approach test}

Social interaction tests can be used to measure anxiety-like behaviours in animals, with a reduction in time spent in social interaction indicating an anxiogenic treatment effect (File \& Seth, 2003). The three-chamber test used here is a test of sociability designed to measure social deficits relevant to autism (Yang et al., 2011), but it is also relevant to depression and other psychiatric disorders characterized by impaired social behaviours (Kaidanovich-Beilin et al., 2011). In many tests of social interaction, both the test subject and social target are allowed to move freely and interact with one another. However the three-chamber design used in this experiment contained the social target within a small cage, preventing physical contact between the test subject and social target. This is advantageous because only the test subject can initiate and control interactions, and fighting or aggressive interactions are avoided. The test subject can also choose to remain more distantly in the empty chamber or with the novel object, reducing the overall stress level of this test (Kaidanovich-Beilin et al., 2011). This test is sensitive to the anxiety-promoting or reducing effects of an experimental treatment. Many benzodiazepines are effective in promoting sociability in the social interaction test, whereas antidepressants are less reliable in having an anxiolytic effect (File \& Seth, 2003).

In the present experiment, few measures attained statistical significance in social approach testing. Stressor-exposed animals did spend significantly less time interacting with a social target (Fig. 2C), although socializing was still present in all treatment groups (Fig. 2D), which may bring the sufficiency of our stressor paradigm into question. No other measures reached statistical significance, although the effects of EPO treatments came close to significantly increasing test subjects' latencies to interact with a social target (Fig. 2B). 
As mentioned previously, sociability in this test can be defined as spending significantly more time in the chamber containing the social target compared to the chamber containing the novel object (Kaidanovich-Beilin et al., 2011). It's worth asking whether or not our stressor paradigm was sufficiently stressful to induce social deficits, given that stressor-exposed animals still spent more time in the chamber containing a social target compared to the chamber containing a novel object. Although few other papers have used the three-chamber test to assess stressor-induced social deficits, Van der Kooij et al. (2013) used the same three-chamber social approach test as reported here. These researchers also found that time spent interacting with a social target was reduced by stressor exposure, whereas the time spent interacting with a novel object was unaffected. Although it was not directly reported in their paper, it did appear that both stressor-exposed and control animals spent considerably more time interacting with the social target compared to the novel object, so that sociability was not necessarily affected by all definitions of this behaviour. The stressor paradigm used by Van der Kooij et al. (2013) involved 21 days of restraint, for 6 hours per day in wire mesh restrainers measuring $26 \mathrm{~cm} \times 26$ $\mathrm{cm}$. Given the relatively large dimensions of the restraint apparatus and the potential for animals to adapt to the stressor over the course of three weeks, the stressor paradigm used in our experiment was arguably stronger than the paradigm used by these experimenters. Still, Van der Kooij et al. (2013) also observed weight reductions and increased aggression during a residentintruder test as a result of stressor treatments, in addition to synaptic changes in the hippocampus. In light of this, it could be argued that the stressor paradigm used in the present study was sufficiently strong to induce some features of social avoidance. It seems that animals can spend significantly more time with a social target compared to a novel object, but still experience some social deficits and hippocampal alterations relevant to depression. This idea is 
supported by Eagle et al., (2013) who assessed social approach in a three-chamber test after rats were exposed to a single prolonged stressor that included 2 hours of restraint, 20 minutes of a forced swim, and diethyl ether exposure. It was observed that both control and stressor-exposed animals spent significantly more time with a social target than an empty basket in the threechambered social approach test. However in more extensive testing, stressor-exposed animals did possess impaired preference for social and object novelty as well as impaired behavioural flexibility. Based on the work of Van der Kooij et al. (2013) and Eagle et al. (2013), it seems that even if animals show a preference for a social target over a novel object, other social deficits may still be present. In our results, stressor-exposed animals spent significantly less time interacting with a social target compared to control animals, which may sufficiently indicate social avoidance in the stressor-exposed animals.

EPO did not reverse any social deficits caused by stressor exposure in this experiment (Fig. 2 C). In other behavioural measures of anxiety, EPO was variably successful in reducing anxiety-like behaviours. In the novelty induced hypophagia test in healthy rats, EPO did significantly reduce the latency to feed in a brightly lit novel environment, indicating an anxiolytic effect (Girgenti et al., 2009). This testing occurred four hours after the final of four daily EPO injections.

However, in mice, EPO did not have any beneficial effects in a light/dark transition test (Leconte et al., 2011). In this case, testing occurred in healthy mice after 5 weeks of twice-weekly EPO injections. Similarly, when healthy mice were given EPO injections every other day for 3 weeks (11 injections in total), there were no effects of the treatment on behaviour in the open field test or elevated plus maze (Adamcio et al., 2008). However, a beneficial effect of EPO in these tests may only become apparent when animals are also treated with stressor exposures. For example, healthy and stressor-exposed mice were given thrice weekly injections of EPO or saline for two 
weeks and then tested in the open field test and elevated plus maze (Osborn et al., 2013). In this case, only stressor-exposed, saline-treated mice had impaired performances in these tests of anxiety, indicating the anxiolytic activity of EPO.

Given that our stressor paradigm induced social avoidance, and that EPO has previously been shown to elicit anxiolytic activity in rats and mice, the question remains as to why EPO did not reverse social avoidance in stressor-exposed rats. As EPO improves memory (Miskowiak et al., 2012) and enhances fear conditioning (Adamcio et al., 2008; Miu et al., 2004), it is possible that when EPO is administered during stressor treatment, the effects might not be entirely positive. For example, could EPO treatment enhance an animal's memory of the stressors, or increase stress reactivity? Perhaps this could also explain why, in the present experiment, EPO treatment trended towards increasing the latency to interact with a social target $(\mathrm{p}=0.08)$. Thus, it would be of interest to assess social approach in rats treated with stressors and given EPO during a recovery period to determine whether this would enhance the anxiolytic effects of EPO treatment.

Another factor to consider is the duration of EPO treatment required for effects on social interaction to become apparent. In social interaction testing in mice exposed to social defeat for ten days, chronic (28 day) treatment with imipramine or fluoxetine, but not acute (one-time) treatment, reversed avoidant behaviour (Berton et al., 2006; Tsankova et al., 2006). In fact, established antidepressants given acutely typically have anxiogenic effects in social interaction testing (File \& Seth, 2003). Perhaps a longer EPO treatment period is needed before the anxiolytic effects are apparent in this particular form of anxiety testing. 
Additionally, the timing of drug administration and behavioural testing may not have been appropriate. In our study, animals were given the final EPO injection two days prior to social interaction testing. In other studies which tested the behavioural effects of EPO in rodents, there was a shorter delay between drug administration and testing. For example, EPO reduced immobility in the force swim test in rats when behavioural testing occurred four hours after the last of four daily injections (Girgenti et al., 2009). Furthermore, a maximum concentration of EPO in brain tissue is reached 10 hours after IP injection in rat pups (Statler et al., 2007).

\section{Changes in growth factor expression in relation to stressor conditions}

Changes in the expression of growth factors measured in this study did not match what was predicted to occur based on other literature. It was predicted that stressor exposure would reduce the expression of BDNF in the hippocampus, since both acute and chronic stressor exposure resulted in reduced BDNF expression in the hippocampus in a number of species (Duman, 2004; Yu \& Chen, 2011). However, in the present study there was no indication of any significant change in hippocampal mRNA expression of BDNF as a result of stressor exposure. As well, in other studies, BDNF mRNA was elevated in the PFC and the amygdala as a result of chronic stressor exposure (Fanous et al., 2010), yet in our study stressor exposure led to a reduction in BDNF mRNA in the PFC, and did not significantly affect expression in the amygdala. As for FGF-2, previous research demonstrated an increase in mRNA levels in the hippocampus and a reduction in the PFC as a result of chronic stress (Fumagalli, et al., 2005) so it was predicted that these effects might be replicated. However, no change was seen in the expression of FGF-2 in the present study in the hippocampus or PFC. In the amygdala, stressor exposure led to a reduction in FGF-2 expression in saline treated animals. 
The lack of change in BDNF expression in the hippocampus may have been a matter of timing. Previously, hippocampal BDNF mRNA in rats was reduced following a single or repeated (7 days) restraint lasting 2 hours each day (Smith et al., 1995). This study found that the reduction in BDNF expression was greatest immediately following the stressor exposure, but intermediate when tissue collection occurred 24 hours after the final stressor (Smith et al., 1995). In another study, BDNF mRNA levels were reduced in the dentate gyrus of rats 0,2 , and 24 hours following an acute, controllable stressor exposure, but not 72 hours after the stressor (Bland et al., 2007). In the present investigation, tissue collection occurred 48 hours after the final stressor exposure, so this time delay could be why chronic stressor exposure appeared not to affect BDNF mRNA levels. Yet, a reduction in hippocampal BDNF protein level was observed several days after the termination of chronic stressor exposure (Mao et al., 2014). This could be reflective of temporal differences in the effects of stressors on mRNA expression versus protein content.

Another consideration in the present investigation concerns whether or not the nonstressor-exposed animals were actually free from stressors. For one thing, the animals in the present experiment were singly housed for the purpose of tracking individual animals' sucrose and water consumptions. However, social isolation may be a chronic stressor, leading to reduced levels of BDNF mRNA in the hippocampus of rats (Adzic et al., 2009). Thus, isolated housing could have been a stressor for all animals in our study. In contrast, in other studies rats were housed in groups (Luoni et al., 2014; Mao et al., 2014; Qui et al., 2014; Roceri et al., 2004), so perhaps the results of this experiment would have been different had we followed that method. 
As well, the animals in the present experiment lacked cage enrichment. BDNF expression is activity-dependent and regulated in part by environmental stimuli (Duman \& Monteggia, 2006). For example, when comparing rats raised for one year in isolated conditions versus an enriched environment, the animals raised in isolation had significantly lower levels of BDNF protein in a number of brain regions, including the cerebral cortex and hippocampal formation (Ickes et al., 2000). Hence, the lack of enrichment in the present experiment may have been another factor which negatively influenced BDNF expression in all animals.

As another point of interest, growth factor analysis was performed on the same animals which received behavioural testing in this experiment. This was done to maximize the use of resources and to be able to correlate growth factor mRNA to behavior. However, behavioural testing can be inherently stressful and thus might influence growth factor mRNA. For example, forced swimming is a behavioural test which has also been used as an acute stressor, variably causing changes in BDNF mRNA levels in the hippocampus and PFC (Fumagalli et al., 2012; Luoni et al., 2014; Shi et al., 2010). It is equally possible that in the present study behavioral testing might have influenced growth factor expression in all animals and thus obscured the effects of the treatments. Finally, the animals used in this study carried a staphylococcus bacterial infection. Although this is common in rodents, the infection might have caused the appearance of skin rashes which were present in some of the control animals. Infection and skin rashes would suggest the presence of inflammation, and inflammatory factors can have neurochemical effects that match those of physical and psychological stressors, including alterations of BDNF expression (Anisman, 2008). In summary, there were a number of unintended stressors to which all of the animals in this study were exposed, which may have impacted subsequent growth factor analyses. 
In the current experiment, BDNF reduction in the PFC as a result of stressor exposure was also not expected. In other research, BDNF mRNA was increased in the PFC following acute or repeated stressors (Bland et al., 2007; Fanous et al., 2010). However, the reduction in mRNA levels in the PFC is not entirely unprecedented. For example, following 2 weeks of twice daily swim stress, chronically stressed rats had BDNF mRNA levels that were approximately $60 \%$ of the control animals' levels (Roceri et al., 2004). BDNF content was also reduced in the frontal cortex of rats that received five weeks of chronic unpredictable mild stress followed by behavioural testing in the forced swim test (Mao et al., 2014),. It seems that there is disagreement in the literature regarding the effects of chronic stress on BDNF expression in the PFC.

A number of problems have been identified in the present experiment, yet the question still remains as to why the hippocampus versus PFC returned such different results with respect to $\mathrm{BDNF}$ mRNA expression. A reduction in hippocampal BDNF mRNA was expected as a result of chronic stressor exposure, yet no change was seen; an elevation was predicted to occur in the PFC, but a large reduction was observed. Given the pitfalls already mentioned, a point worth expanding on is the idea that the behavioural testing acted as an acute stressor in these animals. For example, in other research, animals chronically exposed to a stressor had reduced BDNF mRNA in the hippocampus, but chronic stressor exposure followed by a new acute stressor actually led to normalized BDNF mRNA levels (Adzic et al., 2009). The PFC, in contrast, had BDNF mRNA levels which were significantly reduced in a combined stress group (chronic + acute stressor exposure) relative to the acutely stressed group (Adzic et al., 2009). If our control group is viewed as being acutely stressed as a result of behavioural testing, and our chronically stressed animals are labelled as the "combined stress" group (chronically stressed 
and then acutely stressed with behavioural testing), then the stressor effects on mRNA expression of BDNF in the hippocampus and PFC match the findings of Adzic et al. (2009). The caveat to this explanation is the fact that Adzic et al. (2009) used isolation housing as a chronic stressor - something that all of our animals experienced. Yet in keeping with this idea, when Roceri et al. (2004) observed reduced PFC mRNA levels of BDNF following a chronic swim stressor as mentioned above, this occurred after animals were also briefly restrained and their tails nicked for blood collection prior to sacrifice. In this case the blood collection procedure could have served as an acute, heterotypic stressor, lending support to this argument. Unfortunately the effects of these procedures on hippocampal mRNA levels were not reported in this paper (Roceri et al., 2004).

There was no effect of stress on BDNF expression in the amygdala. Again, the timing of tissue collection and stressor status of control animals may be factors which influenced these results. Additionally, although an increase in BDNF mRNA was previously observed in the amygdala following repeated social defeat stress (Fanous et al., 2010), this effect is not always observed. Several studies found no alteration in BDNF mRNA expression in the amygdala following chronic stressor exposure (Allman et al., 2008; Reagan et al., 2007). Curiously, hippocampal levels of BDNF mRNA were also unaffected by chronic stressor treatment in these studies, and it was suggested that BDNF alterations may be an effect seen following stressors of intermediate duration (1-10 days) but not following 3 weeks of stressors (Reagan et al., 2007).

For FGF-2, no changes were seen in mRNA expression in the hippocampus or PFC as a result of stress in this experiment. Previously, FGF-2 mRNA was elevated in the hippocampus and reduced in the PFC following repeated restraint stressors (Fumagalli et al., 2005), although 
the tissue collection in that experiment occurred 6 hours after stressor termination. In contrast, the current study collected brain tissue 2 days after the final stressor was applied, and this difference in timing may explain why no change was seen in FGF-2 mRNA content as a result of stressor treatments. Indeed, the timeframe for mRNA changes following an acute stressor revealed hippocampal elevations in FGF-2 levels 2 and 24 hours following the stressor, but not 72 hours after stressor administration (Bland et al, 2007). Similarly, when rats were injected once with the synthetic glucocorticoid dexamethasone, FGF-2 mRNA was increased in the cortex and hippocampus at 3, 6, or 10 hours following the injection, but not 1 or 24 hours after the injection (Mocchetti et al., 1996). Although the last two examples involved acute rather than chronic insults, it is possible that the termination of stressor administration and tissue collection was ill-timed in our experiment.

In contrast to the lack of change in the hippocampus and PFC, stressor exposure reduced FGF-2 in the amygdala of saline-treated animals. No other papers were found with which these results could be compared. However, it is interesting that the stressor-induced reduction in FGF2 expression was reversed by EPO treatment.

\section{Changes in growth factor expression in relation to EPO treatment}

It was predicted that erythropoietin would generally lead to elevations in the expression of BDNF and FGF-2 mRNA in the brain regions examined. In fact, however, there were no significant changes in either of these growth factors in the hippocampus. In the PFC there was a reduction in BDNF mRNA content as a result of drug treatment, while levels of FGF-2 did not change. Finally, in the amygdala, EPO treatment elevated BDNF expression regardless of stressor status, and also increased FGF-2 expression in stressor-exposed animals. 
In light of these unexpected results, one question to consider is whether or not the dose of EPO used in this study was appropriate. Animals were given approximately $5,000 \mathrm{U}$ of EPO/kg on four separate occasions, with injections occurring every third day. This dose was used in stroke research using rats (Brines et al., 2000; Chang et al., 2005; Mengozzi et al., 2012) and is comparable to the doses used in another stress study (Pawar et al., 2014). However, it is worth elaborating that Pawar et al. (2014) evaluated chronically stressed rats in a rotarod test after ten days of subcutaneous (SC) EPO injections administered every other day. Injections occurred at doses of 1,000, 2,000, and 4,000 IU/kg. Although all doses were effective in increasing rotarod performance, $1,000 \mathrm{IU} / \mathrm{kg}$ was most effective in this regard, and higher doses were considerably less effective. The present study used IP injections to deliver EPO. Notably, IP delivery leads to greater and more rapid increases in plasma levels of EPO compared to SC delivery, and also to higher levels of EPO appearing in brain extracts (Statler et al., 2007). It is conceivable that the IP dose used here was higher than an optimal dose for producing antidepressant-like effects. In another study, an IP dose of 500IU/kg was given to rats leading to elevated BDNF in the dentate gyrus and reduced immobility in a forced swim test (Girgenti et al., 2009), again pointing to a lower dose range being more appropriate when assessing the antidepressant-like effects of EPO in rats.

Another key consideration is the timing of tissue collection relative to drug treatments. In one instance, when rats were subject to a middle cerebral artery occlusion (MCAO) and EPO administration $(5,000 \mathrm{U} / \mathrm{kg}$, IP) one hour later, BDNF mRNA expression was elevated beyond the effects of MCAO alone 6 hours after the MCAO (Mengozzi et al., 2012). In another example, rats were sacrificed 24 hours after the final of 4 consecutive daily IP injections of EPO (500U/kg). In this case, BDNF mRNA was elevated in the hippocampus, although this was only 
apparent in the dentate gyrus and was limited to $15 \%$ elevation beyond control levels (Girgenti et al., 2009). In the current study, tissue collection occurred following behavioural testing, meaning that the final EPO injection occurred a full 3 days prior to tissue collection. Other research indicates that EPO levels in the brain normalize at 24-hours following a single injection (Statler et al., 2007) so the time delay in our study may not have been optimal for observing the short-term effects of EPO injections on growth factor levels. In addition, administering either daily injections of EPO or else employing a longer treatment period may have been more effective, since these were also features of studies which demonstrated antidepressant-like effects of EPO in rats (Girgenti et al., 2009; Pawar et al., 2014).

Surprisingly, amygdala levels of BDNF were elevated by EPO treatment. However, mRNA purity was low for samples from this brain region, so these results should be considered with caution. Still, by comparison, the antidepressant tianeptine increased BDNF in the amygdala, and this may be related to the ability of tianeptine to inhibit stress-induced morphological changes in the hippocampus and amygdala (Reagan et al., 2007). In this way, elevation of BDNF in the amygdala could represent an antidepressant-like effect of EPO. Amygdala expression of FGF-2 was also increased as a result of EPO treatment in stressorexposed animals. It is interesting that in fear conditioning experiments, bilateral infusions of FGF-2 into the basolateral amygdala enhanced extinction recall and prevented fear reinstatement, indicating that elevated FGF-2 in this brain region could have anxiolytic effects (Graham \& Richardson, 2011).

This said, elevated growth factor levels in the amygdala are not necessarily a good thing. For example, when mice were classified as susceptible or resilient following a social defeat 
paradigm, exposing susceptible mice to foot shock paired with an auditory cue led to a two-fold increase in BDNF expression in the basolateral amygdala, but only a weak increase occurred in resilient mice (Krishnan, 2014). Furthermore, local deletion of BDNF in the basolateral amygdala produced mice which were resilient in response to social defeat (Krishnan, 2014). Perhaps enhanced plasticity in the amygdala (elevated FGF-2 or BDNF) could influence the response to environmental stimuli. So, while this plasticity may augment the effects of extinction training following fear conditioning, it also seems to correlate with responsiveness to social defeat. In other words, enhanced plasticity in this brain region could be good or bad depending on exposure to various environmental stimuli.

\section{Conclusion}

In summary, few of the results in this experiment turned out as predicted. There were a number of problems with the design of this experiment. Key concerns surrounded the stressor exposure of control animals (e.g. lack of enrichment, isolation housing), the dose of EPO used, the timing of tissue collection relative to stressor termination and drug treatments, and mRNA sample purity. These factors should have aligned with other relevant literature and the fact that they did not is unfortunate. 


\section{References}

Adamcio, B., Sargin, D., Stradomska, A., Medrihan, L., Gertler, C., Theis, F.,... Ehrenreich, H. (2008). Erythropoietin enhances hippocampal long-term potentiation and memory. BMC Biology, doi:10.1186/1741-7007-6-37

Adzic, M., Djordjevic, J., Djordjevic, A., Niciforovic, A., Demonacos, C., Radojcic, M., \& Krstic-Demonacos, M. (2009). Acute or chronic stress induce cell compartment-specific phosphorylation of glucocorticoid receptor and alter its transcriptional activity in Wistar rat brain. Journal of Endocrinology, 202: 87-97.

Allaman, I., Papp, M., Kraftsik, R., Fiumelli, H., Magistretti, P.J., \& Martin, J.L. (2008).

Expression of brain-derived neurotrophic factor is not modulated by chronic mild stress in the rat hippocampus and amygdala. Pharmacological Reports, 60: 1001-1007.

American Psychological Association. (2014). Depression. Retrieved from: http://www.apa.org/topics/depress/index.aspx

Anisman, H., 2008. Cascading effects of stressors and inflammatory immune system activation: implications for major depressive disorder. Journal of Psychiatry and Neuroscience, 34: 4-20.

Audet, M.C., Jacobson-Pick, S., Wann, B.C., \& Anisman, H. (2011). Social defeat promotes specific cytokine variations within the prefrontal cortex upon subsequent aggressive or endotoxin challenges. Brain, Behavior, and Immunity, 25: 1197-1205.

Baker, S.L., Kentner, A.C., Konkle, A.T.M., Santa-Maria Barbagallo, L., \& Bielajew, C. (2006). Behavioral and physiological effects of chronic mild stress in female rats. Physiology \& Behavior, 87: $314-322$.

Bekris, S., Antoniou, K., Daskas, S., \& Papadopoulou-Daifoti, Z. (2005). Behavioural and neurochemical effects induced by chronic mild stress applied to two different rat strains. Behavioural Brain Research, 161: 45-59.

Berton, O., McClung, C.A., DiLeone, R.J., Krishnan, V., Renthal, W., Russo, S.J.,...\& Nestler, E.J. (2006). Essential role of BDNF in the mesolimbic dopamine pathway in social defeat stress. Science, 311: 864 - 868.

Bessa, J.M., Morais, M., Marques, F., Pinto, L., Palha, J.A., Almeida, O.F.X., \& Sousa, N. (2013). Stress-induced anhedonia is associated with hypertrophy of medium spiny neurons of the nucleus accumbens. Translational Psychiatry, doi:10.1038/tp.2013.39

Bland, S.T., Tamlyn, J.P., Barrientos, R.M., Greenwood, B.N., Watkins, L.R., Campeau, S., \& Maier, S.F. (2007). Expression of fibroblast growth factor-2 and brain-derived neurotrophic factor mRNA in the medial prefrontal cortex and hippocampus after uncontrollable or controllable stress. Neuroscience, 144: 1219-1228. 
Branchi, I. (2011). The double edged sword of neural plasticity: Increasing serotonin levels leads to both greater vulnerability to depression and improved capacity to recover.

Psychoneuroendocrinology, 36: 339-351.

Brines, M.L., Ghezzi, P., Keenan, S., Agnello, D., de Lanerolle, N.C., Cerami, C., ...\& Cerami, A. (2000). Erythropoietin crosses the blood-brain barrier to protect against experimental brain injury. Proceedings of the National Academy of Sciences, 97: 10526-10531.

Byts, N., \& Sirén, A.L. (2009). Erythropoietin: a multimodal neuroprotective agent.

Experimental \& Translational Stroke Medicine, doi:10.1186/2040-7378-1-4

Castrén, E., Võikar, V., \& Rantamäki, T. (2007). Role of neurotrophic factors in depression. Current Opinion in Pharmacology, 7: 18-21.

Chang, Y.S., Mu, D., Wendland, M., Sheldon, R.A., Vexler, Z.S., McQuillen, P.S., \& Ferriero, D.M. (2005). Erythropoietin improves functional and histological outcome in neonatal stroke. Pediatric Research, 58: 106-111.

Cheeta, S., Broekkamp, C., \& Willner, P. (1994). Stereospecific reversal of stress-induced anhedonia by mianserin and its (+)-enantiomer. Psychopharmacology, 116 : 523-528.

Chen, M., \& Russo-Neustadt, A. (2013). Kinetics of norepinephrine- and serotonin-induced BDNF release in cultured embryonic hippocampal neurons. Neuroscience \& Medicine, 4: 194207.

Colla, M., Kronenberg, G., Deuschle, M., Meichel, K., Hagen, T., Bohrer, M., \& Heuser, I. (2007). Hippocampal volume reduction and HPA-system activity in major depression. Journal of Psychiatric Research, 41: 553-560.

Diorio, D., Viau, V., \& Meaney, M.J. (1993). The role of the medial prefrontal cortex (cingulate gyrus) in the regulation of hypothalamic-pituitary-adrenal responses to stress. The Journal of Neuroscience, 13: 3839-3847.

Duman, R.S. (2014). Neurobiology of stress, depression, and rapid acting antidepressants: remodelling synaptic conections. Depression and Anxiety, 31: 291-296.

Duman, R.S. (2004). Role of neurotrophic factors in the etiology and treatment of mood disorders. NeuroMolecular Medicine, 5: 11-25.

Duman, R.S., Malberg, J., \& Thome, J. (1999). Neural plasticity to stress and antidepressant treatment. Biological Psychiatry, 46: 1181-1191.

Dwivedi, Y., Rizawi, H.S., \& Pandey, G.N. (2006). Antidepressants reverse corticosteronemediated decrease in BDNF expression: dissociation in regulation of specific exons by antidepressants and corticosterone. Neuroscience, 139: 1017-29. 
Eagle, A.L., Fitzpatrick, C.J., \& Perrine, S.A. (2013). Single prolonged stress impairs social and object novelty recognition in rats. Behavioural Brain Research, 256: 591- 597.

Evans, S.J., Choudary, P.V., Neal, C.R., Li, J.Z., Vawter, M.P., Tomita, H., ...\& Akil, H. (2004). Dysregulation of the fibroblast growth factor system in major depression. Proceedings of the National Academy of Sciences, 101: 15506-15511.

Fanous, S., Hammer, R.P., \& Nikuilna, E.M. (2010). Short- and long-term effects of intermittent social defeat stress on BDNF expression in mesocorticolimbic brain regions. Neuroscience, 167: 598-607.

File, S.E. \& Seth, P. (2003). A review of 25 years of the social interaction test. European Journal of Pharmacology, 463: 35- 53.

Forbes, N.F., Stewart, C.A., Matthews, K., \& Reid, I.C. (1996). Chronic mild stress and sucrose consumption: Validity as a model of depression. Physiology and Behavior, 60: 1481-1484.

Fumagalli, F., Bedogni, F., Slotkin, T.A., Racagni, G., \& Riva, M.A. (2005). Prenatal stress elicits regionally selective changes in basal FGF-2 gene expression in adulthood and alters the adult response to acute or chronic stress. Neurobiology of Disease, 20: 731-737.

Fumagalli, F., Calabrese, F., Luoni, A., Shahid, M., Racagnia, G., \& Riva, M.A. (2012). The AMPA receptor potentiator Org 26576 modulates stress-induced transcription of BDNF isoforms in rat hippocampus. Pharmacological Research, 65: 176- 181.

Gaughran, F., Payne, J., Sedgwick, P.M., Cotter, D., \& Berry, M. (2006). Hippocampal FGF-2 and FGFR1 mRNA expression in major depression, schizophrenia and bipolar disorder. Brain Research Bulletin, 70: 221-227.

Girgenti, M.J., Hunsberger, J., Duman, C.H., Sathyanesan, M, Terwilliger, R., \& Newton, S.S. (2009). Erythropoietin induction by electroconvulsive seizure gene regulation, and antidepressant-like behavioral effects. Biological Psychiatry, 66: 267-274.

Graham, B.M., \& Richardson, R. (2011). Intraamygdala infusion of fibroblast growth factor 2 enhances extinction and reduces renewal and reinstatement in adult rats. The Journal of Neuroscience, 31:14151-14157.

Grønli, J., Murison, R., Fiske, E., Bjorvatn, B., Sørensen, E., Portasa, C.M., \& Ursin, R. (2005). Effects of chronic mild stress on sexual behavior, locomotor activity and consumption of sucrose and saccharine solutions. Physiology \& Behavior, 84: 571-577.

Guilloux, J.P., Douillard-Guilloux, G., Kota, R., Wang, X., Gardier, A., Martinowich, K.,... Sibille, E. (2012). Molecular evidence for BDNF- and GABA-related dysfunctions in the amygdala of female subjects with Major Depression. Molecular Psychiatry, 17: 1130-1142. 
Hart, P.C., Bergner, C.L., Dufour, B.D., Smolinsky, A.N., Egan, R.J., LaPorte, J.L., \& Kalueff, A.V. (2009). Analysis of abnormal repetitive behaviours in experimental animal models. In Translational Neuroscience (chapter 4). Retrieved from: http://www.kaluefflab.com/pdfs/chapters/TransNeuroAdvAnimalResearchEthics(Hart\&AVK).p df

Hayley, S., Du, L., Littlejohn, D., Palkovits, M., Faludi, G., Merali, Z., ... Anisman, H. (2014). Brain derived neurotrophic factor is differentially expressed within the frontal cortex and hippocampus of depressed males and females that died through suicide. Department of Neuroscience, Carleton University, Ottawa, Ontario, Canada. Manuscript in preparation.

Hayley, S., \& Littlejohn, D. (2013). Neuroplasticity and the next wave of antidepressant strategies. Frontiers in Cellular Neuroscience, doi: 10.3389/fncel.2013.00218

Hoon Oh, D., Lee, I.Y., Choi, M., Kim, S.H., \& Son, H. (2012). Comparison of neurite outgrowth induced by erythropoietin (EPO) and carbamylated erythropoietin (CEPO) in hippocampal neural progenitor cells. Korean Journal of Physiology and Pharmacology, 16: 281285.

Ickes, B.R., Pham, T.M., Sanders, L.A., Albeck, D.S. Mohammed, A.H. \& Granholm, A.C. (2000). Long-term environmental enrichment leads to regional increases in neurotrophin levels in rat brain. Experimental Neurology, 164: 45-52.

Kaidanovich-Beilin, O., Lipina, T., Vukobradovic, I., Roder, J., \& Woodgett, J.R. (2011). Assessment of social interaction behaviors. Journal of Visualized Experiments, doi: $10.3791 / 2473$

Karege, F., Vaudana, G., Schwalda, M., Perrouda, N., \& LaHarpe, R. (2005). Neurotrophin levels in postmortem brains of suicide victims and the effects of antemortem diagnosis and psychotropic drugs [Abstract]. Molecular Brain Research, 136: 29-37.

Kobayashia, K., Ikeda, Y., Sakaia, A., Yamasaki, N., Haneda, E., Miyakawab, T., \& Suzuki, H. (2010). Reversal of hippocampal neuronal maturation by serotonergic antidepressants.

Proceedings of the National Academy of Sciences, 107: 8434-8439.

Koegh, C.L., Yu, S.P. \& Wei, L. (2007). The effect of recombinant human erythropoietin on neurovasculature repair after focal ischemic stroke in neonatal rats. The Journal of Pharmacology and Experimental Therapeutics, 322: 521-528.

Konkle, A.T.M., Baker, S.L., Kentner, A.C., Santa-Maria Barbagallo, L., Merali, Z., \& Bielajew, C. (2003). Evaluation of the effects of chronic mild stressors on hedonic and physiological responses: sex and strain compared. Brain Research, 992: 227- 238.

Krishnan, V. (2014). Defeating the fear: New insights into the neurobiology of stress susceptibility. Experimental Neurology, 261: 412-416. 
Leconte, C., Bihel, E., Lepelletier, F.X., Bouët, V., Saulnier, R., Petit, E., ... Schumann-Bard, P. (2011). Comparison of the effects of erythropoietin and its carbamylated derivative on behaviour and hippocampal neurogenesis in mice. Neuropharmacology, 60: 354-364.

Liston, C., Miller, M.M., Goldwater, D.S., Radley, J.J., Rocher, A.B., Hof, P.R., ... McEwen, B.S. (2006). Stress-induced alterations in prefrontal cortical dendritic morphology predict selective impairments in perceptual attentional set-shifting. The Journal of Neuroscience, 26: $7870-7874$.

Livak, K.J., \& Schmittgen, T.D. (2001). Analysis of relative gene expression data using realtime quantitative PCR and the $2^{-\Delta \Delta C}$ method. Methods, 25: 402-408.

Luoni, A., Fumagalli, F., Racagni, G., Riva, M.A. (2014). Repeated aripiprazole treatment regulates Bdnf, Arc and Npas4 expression under basal condition as well as after an acute swim stress in the rat brain. Pharmacological Research, 80: 1-8.

MacQueen, G.M., Campbell, S., McEwen, B.S., Macdonald, K., Amano, S., Joffe, R.T., ... Young, L.T. (2003). Course of illness, hippocampal function, and hippocampal volume in major depression. Proceedings of the National Academy of Sciences, 100: 1387-1392.

Maheu, M.E., Davoli, M.A., Turecki, G., \& Mechawar, N. (2013). Amygdalar expression of proteins associated with neuroplasticity in major depression and suicide. Journal of Psychiatric Research, 47: 384-390.

Malberg, J.E. (2004). Implications of adult hippocampal neurogenesis in antidepressant action. Journal of Psychiatry and Neuroscience, 29: 196-205.

Mallei, A., Shi, B., \& Mocchetti, I. (2002). Antidepressant treatments induce the expression of basic fibroblast growth factor in cortical and hippocampal neurons. Molecular Pharmacology, 61: $1017-1024$.

Mao, Q.Q., Huang, Z., Zhong, X.M., Xian, Y.F., \& Ip, S.P. (2014). Piperine reverses chronic unpredictable mild stress-induced behavioral and biochemical alterations in rats. Cellular and Molecular Neurobiology, 34: 403-408.

Maragnoli, M.E., Fumagalli, F., Gennarelli, M., Racagni, G., \& Riva, M.A. (2004). Fluoxetine and olanzapine have synergistic effects in the modulation of fibroblast growth factor 2 expression within the rat brain. Biological Psychiatry, 55: 1095-1102.

Masi, G., \& Brovedani, P. (2011). The Hippocampus, neurotrophic factors and depression: Possible implications for the pharmacotherapy of depression. CNS Drugs, 25: 913-931.

McEwen, B.S. (2004). Structural plasticity of the adult brain: how animal models help us understand brain changes in depression and systemic disorders related to depression. Dialogues in Clinical Neuroscience, 6: 119-133. 
Mengozzi, M., Cervellini, I., Villa, P., Erbayraktar, Z., Gökmen, N., Yilmaz, O., ...\& , P. (2012). Erythropoietin-induced changes in brain gene expression reveal induction of synaptic plasticity genes in experimental stroke. Proceedings of the National Academy of Sciences, 109: 96179622.

Mirescu, C. \& Gould, E. (2006). Stress and Adult Neurogenesis. Hippocampus, 16: 233-238.

Miskowiak, K.W., Favaron , E., Hafizi, S., Inkster, B., Goodwin, G.M., Cowen, P.J., \& Harmer, C.J. (2009). Effects of erythropoietin on emotional processing biases in patients with major depression: an exploratory fMRI study. Psychopharmacology, 207: 133-142.

Miskowiak, K.W., Favaron, E., Hafizi, S., Inkster, B., Goodwin, G.M., Cowen, P.J., \& Harmer, C.J. (2010) Erythropoietin modulates neural and cognitive processing of emotional information in biomarker models of antidepressant drug action in depressed patients. Psychopharmacology, 210: $419-428$.

Miskowiak, K.W., O’Sullivan, U., \& Harmer, C.J. (2007). Erythropoietin reduces neural and cognitive processing of fear in human models of antidepressant drug action. Biological Psychiatry, 62: 1244-1250.

Miskowiak, K.W., Vinberg, M, Harmer, C.J., Ehrenreich, H. \& Kessing, L. (2012). Erythropoietin: a candidate treatment for mood symptoms and memory dysfunction in depression. Psychopharmacology, 219: 687-698.

Miu, A.C., Olteanu, A.I., Chi, I., \& Heilman, R.M. (2004). Have no fear, erythropoietin is here: erythropoietin protects fear conditioning performances after functional inactivation of the amygdala. Behavioural Brain Research, 155: 223-229.

Mocchetti, I., Spiga, G., Hayes, V.Y., Isackson, P.J., \& Colangelo, A. (1996). Glucocorticoids differentially increase nerve growth factor and basic fibroblast growth factor expression in the rat brain. The Journal of Neuroscience, 16: 2141-2148.

Molteni, R., Fumagalli, F., Magnaghi, V., Roceri, M., Gennarelli, M., Racagnia, G., ... Riva, M.A. (2001). Modulation of fibroblast growth factor-2 by stress and corticosteroids: from developmental events to adult brain plasticity. Brain Research Reviews, 37: 249-258.

Moreau, J.L. (2002). Simulating the anhedonia symptom of depression in animals. Dialogues in Clinical Neuroscience, 4: 351-360.

Muscat, R., \& Willner, P. (1992). Suppression of sucrose drinking by chronic mild unpredictable stress: A methodological analysis. Neuroscience and Biobehavioral Reviews, 16: 507-517.

Osborn, M., Rustom, N., Clarke, M., Littlejohn, D., Rudyk, C., Anisman, H., \& Hayley, S. (2013). Antidepressant-like effects of erythropoietin: A focus on behavioural and hippocampal processes. PLoS ONE, 8: e72813. doi:10.1371/journal.pone.0072813 
Papp, M., Willner, P., \& Muscat, R. (1991). An animal model of anhedonia: attenuation of sucrose consumption and place preference conditioning by chronic unpredictable mild stress. Psychopharmacology, 104: 255-259.

Pariante, C.M. (2006). The glucocorticoid receptor: Part of the solution or part of the problem? Journal of Psychopharmacology, 20: 79-84.

Pawar, A.S., Barve, K.H., Patel, A., Raje, A., \& Addepalli, V.A. (2014). A possible mechanism of antidepressant like effect of recombinant human erythropoietin. Journal of Neurology and Neurophysiology, doi:10.4172/2155-9562-5-1000222

Perez, J.A., Clinton, S.M., Turner, C.A., Watson, S.J., \& Akil, H. (2009). A new role for FGF2 as an endogenous inhibitor of anxiety. The Journal of Neuroscience, 29: 6379-6387.

Pezawas, L., Meyer-Lindenberg, A., Goldman1, A.L., Verchinski, B.A., Chen, G., Kolachana, B.S., ...\& Weinberger, D.R. (2008). Evidence of biologic epistasis between BDNF and SLC6A4 and implications for depression. Molecular Psychiatry, 13: 709-716.

Piccinni, A., Marazziti, D., Catena, M., Domenici, L., Del Debbio, A., Bianchi, C., ... Dell'Osso, L. (2008). Plasma and serum brain-derived neurotrophic factor (BDNF) in depressed patients during one year of antidepressant treatments. Journal of Affective Disorders, 105: 279-283.

Pittenger, C. \& Duman, R.S. (2008). Stress, depression, and neuroplasticity: A convergence of mechanisms. Neuropsychopharmacology, 33: 88-109.

Pizarro, J.M., Lumley, L.A., Medina, W., Robison, C.L., Chang, W.E., Alagappan, A.,... Meyerhoff, J.L. (2004). Acute social defeat reduces neurotrophin expression in brain cortical and subcortical areas in mice. Brain Research, 1025: 10-20.

Qui, H.M., Yang, J.X., Liu, D., Fei, H.Z., Hu, X.Y., \& Zhou, Q.X. (2014). Antidepressive effect of sodium valproate involving suppression of corticotropin-releasing factor expression and elevation of BDNF expression in rats exposed to chronic unpredicted stress. NeuroReport, 25: 205-210.

Reagan, L.P., Hendry, R.M., Reznikov, L.R., Piroli, G.G., Wood, G.E., McEwen, B.S., \& Grillo, C.A. (2007). Tianeptine increases brain-derived neurotrophic factor expression in the rat amygdala. European Journal of Pharmacology, 565: 68-75.

Roceri, M., Cirulli, F., Pessina, C., Peretto, P., Racagni, G., Riva, M.A. (2004). Postnatal repeated maternal deprivation produces age-dependent changes of brain-derived neurotrophic factor expression in selected rat brain regions. Biological psychiatry, 55: 708-714.

Saarelainen, T., Hendolin, P., Lucas, G., Koponen, E., Sairanen, M., MacDonald, E.,... Castrén, E. (2003). Activation of the TrkB neurotrophin receptor is induced by antidepressant drugs and is required for antidepressant-induced behavioral effects. The Journal of Neuroscience, 23: 349 357. 
Sandi, C. \& Losertales, M. (1999). Opposite effects on NCAM expression in the rat frontal cortex induced by acute vs. chronic corticosterone treatments. Brain Research, 828: 127-134.

Schmittgen, T.D., \& Livak, K.J. (2008). Analyzing real-time PCR data by the comparative $\mathrm{C}_{\mathrm{T}}$ method. Nature Protocols, 3: 1101-1108.

Shelton, R.C., \& Hollon, S.D. (2012). The long-term management of major depressive disorders. Focus, 10: 434-441.

Shi, S.S., Shao, S.H., Yuan, B.P., Pan, F., \& Li, Z.L. (2010). Acute stress and chronic stress change brain-derived neurotrophic factor (BDNF) and tyrosine kinase-coupled receptor (TrkB) expression in both young and aged rat hippocampus. Yonsei Medical Journal, 51: 661-671.

Smith, M.A., Makino, S., Kvetnansky, R., \& Post, R.M. (1995). Stress and glucocorticoids affect the expression of brain derived neurotrophic factor and neurotrophin-3 mRNA in the hippocampus. The Journal of Neuroscience, 15: 1768-1777.

Statler, P.A., McPherson, R.J., Bauer, L.A., Kellert, B.A., \& Juul, S.E. (2007).

Pharmacokinetics of high-dose recombinant erythropoietin in plasma and brain of neonatal rats. Pediatric Research, 61: 671-675.

Strekalova, T., Couch, Y., Kholod, N., Boyks, M., Malin, D., Leprince, P., \& Steinbusch, H.M.W. (2011). Update in the methodology of the chronic stress paradigm: internal control matters. Behavioral and Brain Functions, doi:10.1186/1744-9081-7-9

Son, H., Banasr, M., Choi, M., Chae, S.Y., Licznerski, P., Lee, B.,...Duman, R.S. (2012). Neuritin produces antidepressant actions and blocks the neuronal and behavioral deficits caused by chronic stress. Proceedings of the National Academy of Sciences, 109: 11378-11383.

Sousa, N., Lukoyanov, N.V., Madeira, M.D., Almeida, O.F.X., \& Paula-Barbosa, M.M. (2000). Reorganization of the morphology of hippocampal neurites and synapses after stress-induced damage correlates with behavioral improvement. Neuroscience, 97: 253-266.

Thermo Scientific. (n.d.). Assessment of nucleic acid purity. Retrieved From: http://www.nanodrop.com/Library/T042-NanoDrop-Spectrophotometers-Nucleic-Acid-PurityRatios.pdf

Thorne, R.G., \& Frey, W.H. (2001). Delivery of neurotrophic factors to the central nervous system: Pharmacokinetic considerations. Clinical Pharmacokinetics, 40: 907-946.

Tsankova, N.M., Berton, O., Renthal, W., Kumar, A., Neve, R.L. \& Nestler, E.J. (2006). Sustained hippocampal chromatin regulation in a mouse model of depression and antidepressant action. Nature Neuroscience, 9: 519-525. 
Valverde, O., Smadja, C., Roques, B.P., \& Maldonado, R. (1997). The attenuation of morphineconditioned place preference following chronic mild stress is reversed by a CCKB receptor antagonist. Psychopharmacology, 131: 79-85.

van der Kooij, M.A., Fantin, M., Kraev, I., Korshunova, I., Grosse, J., Zanoletti, O., ... \& Sandi, C. (2013). Impaired hippocampal neuroligin-2 function by chronic stress or synthetic peptide treatment is linked to social deficits and increased aggression. Neuropsychopharmacology, doi:10.1038/npp.2013.315

Vyas, A., Mitra, R., Shankaranarayana R.B.S., \& Chattarji, S. (2002). Chronic stress induces contrasting patterns of dendritic remodeling in hippocampal and amygdaloid neurons. The Journal of Neuroscience, 22: 6810-6818.

Wellman, C.L. (2001). Dendritic reorganization in pyramidal neurons in medial prefrontal cortex after chronic corticosterone administration. Journal of Neurobiology, 49: 245-253, 2001.

Willner, P. (1997). Validity, reliability and utility of the chronic mild stress model of depression: a 10-year review and evaluation. Psychopharmacology, 134: 319-329.

Willner, P., Towell, A., Sampson, D., Sophokleous, S., \& Muscat, R. (1987). Reduction of sucrose preference by chronic unpredictable mild stress, and its restoration by a tricyclic antidepressant. Psychopharmacology, 93: 358-364.

World Health Organization. (2012). Depression. Retrieved from: http://www.who.int/mediacentre/factsheets/fs369/en/

Yang, M., Silverman, J.L., \& Crawley, J.N. (2011). Automated three-chambered social approach task for mice. Current Protocols in Neuroscience, doi: 10.1002/0471142301.ns0826s56

Yu, H. \& Chen, Z. (2011). The role of BDNF in depression on the basis of its location in the neural circuitry. Acta Pharmacologica Sinica, 32: 3-11.

Zhang, L., Luo, J., Zhang, M., Yao, W., Ma, X., \& Yu, S. (2014). Effects of curcumin on chronic, unpredictable, mild, stress-induced depressive-like behaviour and structural plasticity in the lateral amygdala of rats. International Journal of Neuropsychopharmacology, 17: 793-806.

Zobel, A.W., Nickel, T., Sonntag, A., Uhr, M., Holsboer, F., \& Ising, M. (2001). Cortisol response in the combined dexamethasone/CRH test as predictor of relapse in patients with remitted depression: a prospective study. Journal of Psychiatric Research, 35: 83-94. 